\title{
HR 10: a main-sequence binary with circumstellar envelopes around both components
}

\section{Discovery and analysis ${ }^{\star}$}

B. Montesinos ${ }^{1}$, C. Eiroa ${ }^{2,3}$, J. Lillo-Box ${ }^{4,1}$, I. Rebollido ${ }^{2}$, A. A. Djupvik ${ }^{5}$, O. Absil ${ }^{6, \star \star}$, S. Ertel ${ }^{7,8}$, L. Marion ${ }^{6}$, J. J. E. Kajava ${ }^{9}$, S. Redfield ${ }^{10}$, H. Isaacson ${ }^{11}$, H. Cánovas ${ }^{12}$, G. Meeus ${ }^{2}$, I. Mendigutía ${ }^{1}$, A. Mora ${ }^{13}$, P. Rivière-Marichalar ${ }^{14}$, E. Villaver ${ }^{2}$, J. Maldonado ${ }^{15}$, and T. Henning ${ }^{16}$

\footnotetext{
${ }^{1}$ Departmento de Astrofísica, Centro de Astrobiología (CAB, CSIC-INTA), ESAC Campus, Camino Bajo del Castillo s/n, 28692 Villanueva de la Cañada, Madrid, Spain e-mail: bmm@cab.inta-csic.es

${ }^{2}$ Departamento Física Teórica, Facultad de Ciencias, Universidad Autónoma de Madrid, Campus de Cantoblanco, 28049 Madrid, Spain

${ }^{3}$ Observatorio Astrónomico de Calar Alto, CAHA, 04550 Gérgal, Almería, Spain

${ }^{4}$ European Southern Observatory (ESO), Alonso de Córdova 3107, Vitacura, Casilla 19001, Santiago de Chile, Chile

${ }^{5}$ Nordic Optical Telescope, Apartado 474, 38700 Santa Cruz de La Palma, Santa Cruz de Tenerife, Spain

${ }^{6}$ Space sciences, Technologies, and Astrophysics Research (STAR) Institute, University of Liège, Belgium

${ }^{7}$ Large Binocular Telescope Observatory, 933 North Cherry Avenue, Tucson, AZ 85721, USA

${ }^{8}$ Steward Observatory, Department of Astronomy, University of Arizona, 993 N. Cherry Avenue, Tucson, AZ 85721, USA

${ }^{9}$ Finnish Centre for Astronomy with ESO (FINCA), University of Turku, 20014 Finland

${ }^{10}$ Astronomy Department and Van Vleck Observatory, Wesleyan University, Middletown, CT 06459, USA

${ }^{11}$ Department of Astronomy, University of California, Berkeley, CA, USA

${ }^{12}$ ESA-ESAC, Operations Department, Camino Bajo del Castillo s/n, 28692 Villanueva de la Cañada, Madrid, Spain

${ }^{13}$ Aurora Technology B.V. for ESA, ESA-ESAC, Camino Bajo del Castillo s/n, 28692 Villanueva de la Cañada, Madrid, Spain

${ }^{14}$ Observatorio Astronómico Nacional (OAN-IGN) - Observatorio de Madrid, Alfonso XII, 3, 28014 Madrid, Spain

${ }^{15}$ INAF - Osservatorio Astronomico di Palermo Piazza del Parlamento 1, 90134 Palermo, Italy

${ }^{16}$ Max-Planck-Institut für Astronomie, Königstuhl 17, 69117 Heidelberg, Germany
}

Received 26 June 2019 / Accepted 13 July 2019

\begin{abstract}
Context. This paper is framed within a large project devoted to studying the presence of circumstellar material around main sequence stars, and looking for exocometary events. The work concentrates on HR 10 (A2 IV/V), known for its conspicuous variability in the circumstellar narrow absorption features of $\mathrm{Ca}$ II $\mathrm{K}$ and other lines, so far interpreted as $\beta$ Pic-like phenomena, within the falling evaporating body scenario.

Aims. The main goal of this paper is to carry out a thorough study of HR 10 to find the origin of the observed variability, determine the nature of the star, its absolute parameters, and evolutionary status.

Methods. Interferometric near-infrared (NIR) observations, multi-epoch high-resolution optical spectra spanning a time baseline of more than $32 \mathrm{yr}$, and optical and NIR photometry, together with theoretical modelling, were used to tackle the above objectives.

Results. Our results reveal that HR 10 is a binary. The narrow circumstellar absorption features superimposed on the photospheric Ca II K lines - and lines of other species - can be decomposed into two or more components, the two deep ones tracing the radial velocity of the individual stars, which implies that their origin cannot be ascribed to transient exocometary events, their variability being fully explained by the binarity of the object. There does not appear to be transient events associated with potential exocomets. Each individual star holds its own circumstellar shell and there are no traces of a circumbinary envelope. Finally, the combined use of the interferometric and radial velocity data leads to a complete spectrometric and orbital solution for the binary, the main parameters being: an orbital period of 747.6 days, eccentricities of the orbits around the centre of mass 0.25 (HR 10-A), 0.21 (HR 10-B) and a mass ratio of $q=M_{\mathrm{B}} / M_{\mathrm{A}}=0.72-0.84$. The stars are slightly off the main sequence, the binary being $\sim 530$ Myr old.
\end{abstract}

Key words. binaries: general - circumstellar matter - stars: fundamental parameters - techniques: interferometric techniques: spectroscopic

\footnotetext{
* Partially based on observations obtained with PIONIER/VLT (ESO, Paranal, Chile), FIES/NOT, HERMES/Mercator, HARPS-N/TNG and UES/WHT (La Palma, Spain), FEROS/2.2-m ESO-MPIA (La Silla, Chile), CS21/Harlan J. Smith Telescope (McDonald Observatory, US) and UHRF/3.6-m AAT (Anglo Australian Observatory), and archival data from HARPS/3.6-m ESO and UVES/VLT (ESO archive), and HIRES/Keck 1 (Keck archive).

${ }^{\star \star}$ F.R.S.-FNRS Research Associate.
} 


\section{Introduction}

Once a star has undergone the pre-main sequence phase and the protoplanetary - primordial - disc evolves, experiencing gas dispersal and grain growth (see e.g. Armitage 2015), the surrounding circumstellar (CS hereafter) material, namely comets, minor bodies, dusty debris discs, and gas in shells or envelopes, is as important as planets for providing clues to the early history of the solar system, and by analogy, of exoplanetary systems.

While exoplanets (see e.g. Beuther et al. 2014, and references therein) and debris discs (see e.g. Matthews et al. 2014) are now routinely studied, little is known about comets and other minor bodies around other stars. Their own nature, that is their small size and lack of an atmosphere, makes them elusive to any direct detection, but indirect methods, such as the detection of certain features originated by dust and gas, provide evidence of and information about the presence and properties of this kind of solid-phase CS material.

Dust features offer hints about the properties of $\mu \mathrm{m}$-sized grains in debris discs that result from collisions of planetesimals (e.g. Olofsson et al. 2012). Circumstellar CO emission around some AF-type main sequence (MS) stars (e.g. Moór et al. 2015; Marino et al. 2016) has been interpreted as being the result of outgassing produced by comet collisions (Zuckerman \& Song 2012). A cometary origin of warm (Mennesson et al. 2014; Ertel et al. 2018) and hot (Absil et al. 2006, 2013; Ertel et al. 2014), exozodiacal dust has also been suggested (Faramaz et al. 2017).

On the other hand, spectroscopic monitoring of transient absorption events in CS metallic lines - the Ca II K line being particularly sensitive and prominent (Welsh \& Montgomery 2015) - can provide more direct information on the dynamics and composition of exocomets. The best-known examples are the variable absorption features observed in the spectra of $\beta$ Pic (Hobbs et al. 1985), that are interpreted as the result of gas being released by the sublimation of exocomets grazing or falling onto the star (Ferlet et al. 1987; Kiefer et al. 2014, and references therein), driven into its vicinity by the perturbing action of a larger body, for example a planet. This scenario is the so-called falling evaporating body model (FEB; Beust et al. 1990, 1991). Variable absorption features of this kind have also been observed toward several A-type stars (e.g. Redfield et al. 2007; Roberge \& Weinberger 2008; Welsh \& Montgomery 2015); another example is the discovery and analysis by Grady et al. (2018) of infalling gas in HD 172555, in ultraviolet resonant transitions of Si III and IV, C II and IV, and O I.

Since September 2015 we have been carrying out a highresolution spectroscopic project aiming at detecting and monitoring narrow, variable metallic absorption lines that are attributed to transient exocometary events around MS stars, mainly of A-type. Around 1600 spectra of more than 100 stars have been obtained (Rebollido et al. 2019). The first results of this project are the study of the variations of the CS Ca II HK lines of the A5 V star $\varphi$ Leo (Eiroa et al. 2016), and the co-existence of hot and cold gas in debris discs (Rebollido et al. 2018).

In the context of this project, and in order to contribute to the understanding of the properties of CS material around MS or early post-MS stars, we have focused this work on HR 10 (A2 IV/V), a shell star showing very interesting spectroscopic behaviour linked to its CS material (see Sect. 2). The star has been treated so far as a single object; this fact has biased the interpretation of its observed spectroscopic variability towards scenarios that must be totally revised after the discovery, reported in this paper, that HR 10 is actually a binary.
In Sect. 2 we summarise what is known about this star and relevant to this work; in Sect. 3 we describe and analyse the PIONIER/VLTI observations that lead to the discovery that HR 10 is a binary; in Sect. 4 we describe the high-resolution spectroscopic observations, both from our own campaigns and from archives or the literature, that have been used to characterise the properties and measure the radial velocities $(\mathrm{RVs})$ of the CS narrow absorption features superimposed on the photospheric lines. Section 5 shows details and properties of those CS absorption features. In Sect. 6 we give quantitative details about the RVs, the orbital solution from the interferometric and spectroscopic RV data, and derive the stellar parameters of the individual components of HR 10. In Sect. 7 we present a discussion of the results. Finally, in Sect. 8 we summarise the main conclusions of this work.

\section{Previous work on HR 10}

HR 10 (HD 256, HIP 602) is a bright "shell star" $(V=6.23)$ (Cheng et al. 1991; Jaschek et al. 1991), also labelled as a "disc star" by Abt (2008) under the assumption that due to the large rotation velocity, the CS Ti II lines must originate in a disc. The spectral type classification of the object in the literature varies from A0 to A6, and luminosity classes IV or V. The current most accepted classification is A2 IV/V (see e.g. Wright et al. 2003, VizieR catalogue III/231). The spectrum of HR 10 resembles that of a rapidly rotating early A star $\left(v \sin i=294 \mathrm{~km} \mathrm{~s}^{-1}\right.$, Mora et al. 2001).

Observations taken since the late 1980s show high spectroscopic variability in the narrow absorption components superimposed on the photospheric Ca II K line. Lagrange-Henri et al. (1990) and Welsh et al. (1998) interpreted those variations within the FEB model, which is similar to that devised for $\beta$ Pic.

Redfield et al. (2007) carried out a detailed study of the presence of narrow components superimposed on the photospheric $\mathrm{Ca}$ II and $\mathrm{Na}$ I lines, and their potential short- and long-term variability. The origin of the narrow $\mathrm{Ca}$ II $\mathrm{K}$ absorption was attributed to CS material, with no interstellar contribution, the variability being caused by the closeness of the gas to the star. No significant absorption was detected in $\mathrm{Na}$ I after water vapour lines were removed.

Abt (2008) pointed out the singularity of HR 10 as the only star in a sample of 20 objects analysed showing double Ti II narrow absorptions in the doublet around $376.0 \mathrm{~nm}$. The redshifted components were interpreted as being caused by infalling material onto the star.

Concerning the spectral energy distribution (SED), Cheng et al. (1991) reported a weak IR excess at 12 and $25 \mu \mathrm{m}$ from Infrared Astronomical Satellite (IRAS) data, whereas Redfield et al. (2007) did not detect any significant IR excess in Spitzer Infrared Array Camera (IRAC), Multiband Imaging Photometer (MIPS), or Infrared Spectrograph (IRS) measurements. An upper limit of $L_{\mathrm{IR}} / L_{*}<6.1 \times 10^{-6}$ (consistent with the Spitzer upper limits at the longest IR wavelengths) was given by these latter authors, a value to be compared with $L_{\mathrm{IR}} / L_{*} \simeq 3.0 \times 10^{-3}$ (Backman \& Paresce 1993), or $\simeq 2.4 \times 10^{-3}$ (Heinrichsen et al. 1999) for $\beta$ Pic. There are no traces of accretion or emission lines in the spectra, and therefore the star is most likely not in the pre-MS phase.

So far, HR 10 has been treated in the literature as a single object. In retrospect, this fact has been a tight constraint not only in interpreting the observed variability, but also in determining its absolute parameters and its position in the HR diagram. The single-star assumption yielded some controversial results; the 
Table 1. PIONIER observations of HR 10.

\begin{tabular}{|c|c|c|c|c|}
\hline Date & 2014-09-02 & $2017-09-23$ & 2018-08-07 & 2018-09-05 \\
\hline Starting time [UT] & 06:13:06 & $02: 55: 38$ & 09:15:56 & 07:50:37 \\
\hline BJD [TDB] mid observation & 2456902.7803 & 2458019.6773 & 2458337.8991 & 2458366.8422 \\
\hline Programme & 093.C-0712(B) & 099.C-2015(A) & 0101.C-0182(B) & 0101.C-0182(B) \\
\hline VLTI array & D0-G1-H0-I1 & A0-B2-C1-D0 & A0-G1-J2-J3 & A0-G1-J3-K0 \\
\hline \# SCI observations ${ }^{(a)}$ & 3 & 4 & 2 & 2 \\
\hline Significance in $\mathrm{cp}$ & 1435 & 50 & 283 & 247 \\
\hline Significance in $V^{2}$ & 8271 & 1378 & 1623 & 4948 \\
\hline Significance combined & 1038 & 963 & 1046 & 654 \\
\hline Separation (mas) & $15.98 \pm 0.35$ & $2.94 \pm 0.56$ & $6.34 \pm 0.16$ & $11.43 \pm 0.27$ \\
\hline Position angle (deg) ${ }^{(b)}$ & $39.3 \pm 1.2$ & $77.7 \pm 2.5$ & $32.1 \pm 0.3$ & $37.0 \pm 2.7$ \\
\hline Contrast (\%) & $32.0 \pm 1.5$ & $35 \pm 13$ & $31.9 \pm 3.9$ & $31.9 \pm 2.8$ \\
\hline
\end{tabular}

Notes. ${ }^{(a)}$ Number of observations (observing blocks) executed on the science target and bracketed by observations of calibrators. ${ }^{(b)}$ The position angle is measured east of north, i.e. counterclockwise from the north.
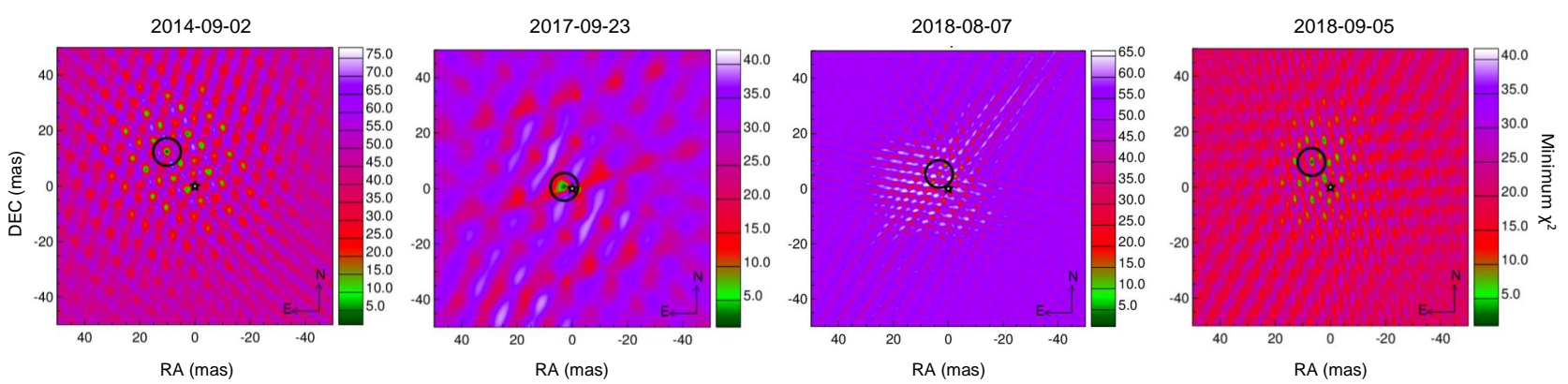

Fig. 1. Normalised $\chi$-square maps of the combined $\mathrm{cp}+V^{2}$ for the four PIONIER/VLTI observations. The maps are centred at the brightest component. The black circles indicate the positions of the minima in the maps and the star marks the position of the bright component.

$V$ magnitude, and the Gaia DR2 distance, $\varpi=6.8882 \pm$ 0.1184 mas, $d=145.18_{-2.45}^{+2.54}$ pc (Gaia Collaboration 2018), respectively, imply an absolute magnitude of $M_{V} \simeq+0.42$, which would put the star closer to luminosity class $\sim \mathrm{III} / \mathrm{IV}$; Redfield et al. (2007) found a value $L_{*} / L_{\odot}=63.8$ which corresponds to a typical $\sim$ A2 III (Aller et al. 1982), both results being in contrast with the spectral classification IV/V. Montesinos et al. (2009) estimated the stellar gravity from the width of the wings of the Balmer lines and derived the mass and age from a $\log g_{*}-\log T_{\text {eff }}$ HR diagram and evolutionary tracks, and then the luminosity from the corresponding point in the $\log L_{*} / L_{\odot}-\log T_{\text {eff }}$ HR diagram; the fact that the Balmer lines - and the whole spectrum are actually the composite of profiles from two stars invalidates the initial measurement of $\log g_{*}$ and then the set of parameters derived. The discovery of the binarity of HR 10 makes a complete reassessment of the stellar parameters compulsory.

\section{PIONIER/VLTI observations}

Interferometric observations of HR 10 were first performed in September 2014 as part of a search for hot exozodiacal dust (see details of the programme in Ertel et al. 2014, 2016) and three more times in September 2017, and August-September 2018, for a total of four epochs. The Precision Integrated Optics Near-Infrared ExpeRiment (PIONIER, Le Bouquin et al. 2011) on the Very Large Telescope Interferometer (VLTI) was used. PIONIER is a four-telescope beam combiner allowing the user to simultaneously obtain squared visibility $\left(V^{2}\right)$ measurements on six baselines and closure phase (cp) measurements on four telescope triplets. A log of the observations is provided in Table 1.
PIONIER operates in $H$ band; the light was dispersed over three (in 2014) and six spectral channels (later) across this band. Chains of alternating observations of a calibration star (CALs) and the science target (SCI) were executed in order to characterise the interferometric transfer function (TF) and calibrate the $V^{2}$ and cp measurements of the science target. Each chain started and ended with a CAL, and various CALs were used within a chain in order to minimise the impact of imperfect knowledge of the calibration stars (e.g. uncertain stellar diameters or unknown companions).

Data reduction and calibration were performed with the PIONIER pipeline pndrs (Le Bouquin et al. 2011) version 3.79 using standard parameters. In particular, we calibrated a whole sequence of CAL and SCI observations at once using the smooth $\mathrm{TF}$ interpolation method provided by the pipeline.

The binary signature is obvious in both the calibrated and uncalibrated $V^{2}$ and cp data, making for a strong detection with each observation. In order to extract the astrometric information (separation and position angle) and contrast (secondary-toprimary flux ratio) of the binary, we follow the method outlined by Absil et al. (2011) and Marion et al. (2014). We compute the $\chi^{2}$ goodness of fit to the $V^{2}$ and cp data, both separately and jointly, for a series of binary star models with a range of positions and flux ratios. The resulting $\chi^{2}$ cubes (one for the $V^{2}$, one for the $\mathrm{cp}$, and one for the combination of both) were used to identify the best-fit model and evaluate the significance of the detection. The results are listed in Table 1. Figure 1 shows the normalised $\chi$-square maps of the combined $\mathrm{cp}+V^{2}$ for the four PIONIER/VLTI observations of HR 10. 
Table 2. Spectroscopic observations of HR 10.

\begin{tabular}{|c|c|c|c|c|c|}
\hline \multicolumn{6}{|c|}{ Dedicated spectroscopic campaigns } \\
\hline Dates & $N$ & Resolution & $\begin{array}{l}\text { Spectral range } \\
\quad(\mathrm{nm})\end{array}$ & $\begin{array}{l}\text { Telescope/Observatory } \\
\text { spectrograph }\end{array}$ & \\
\hline $\begin{array}{ll}2015-09 & 04-07 \\
2015-12 & 20-23 \\
2016-07 & 12\end{array}$ & $\begin{array}{l}17 \\
8 \\
1\end{array}$ & 85000 & $377-900$ & \multicolumn{2}{|c|}{$\begin{array}{l}\text { 1.2-m Mercator Telescope/La Palma } \\
\text { High Efficiency and Resolution Mercator Echelle Spectrograph (HERMES) }\end{array}$} \\
\hline $2015-10 \quad 22-23$ & 3 & 48000 & $350-920$ & \multicolumn{2}{|l|}{$\begin{array}{l}\text { 2.2-m ESO-MPIA Telescope/La Silla } \\
\text { Fibre-fed Extended Range Optical Echelle Spectrograph (FEROS) }\end{array}$} \\
\hline $\begin{array}{ll}2017-10-05 & \text { to } \\
2018-01-03 & \\
2018-11-23 & \text { to } \\
2019-01-08 & \\
\end{array}$ & 10 & 67000 & $370-910$ & \multicolumn{2}{|l|}{$\begin{array}{l}\text { 2.5-m Nordic Optical Telescope (NOT)/La Palma } \\
\text { FIbre-fed Echelle Spectrograph (FIES) }\end{array}$} \\
\hline $\begin{array}{l}2017-10-06 \text { to } \\
2018-01-02\end{array}$ & 21 & 115000 & $383-693$ & \multicolumn{2}{|l|}{$\begin{array}{l}\text { 3.6-m Telescopio Nazionale Galileo (TNG)/La Palma } \\
\text { High Accuracy Radial velocity Planet Searcher North (HARPS-N) }\end{array}$} \\
\hline $\begin{array}{l}2017-07-21 \text { to } \\
2017-12-30\end{array}$ & 21 & $\begin{array}{l}94600 \text { (VIS) } \\
80400 \text { (NIR) }\end{array}$ & $\begin{array}{c}520-960 \\
960-1710\end{array}$ & \multicolumn{2}{|l|}{$\begin{array}{l}\text { 3.5-m CAHA Telescope/Calar Alto } \\
\text { Calar Alto high-Resolution search for M dwarfs with Exoearths } \\
\text { with Near-infrared and optical Echelle Spectrographs (CARMENES) }\end{array}$} \\
\hline \multicolumn{6}{|c|}{ Observations extracted from publications or archives } \\
\hline Dates & $N$ & Resolution & $\begin{array}{l}\text { Spectral range } \\
\text { (line or } \mathrm{nm} \text { ) }\end{array}$ & $\begin{array}{l}\text { Telescope/Observatory } \\
\text { spectrograph }\end{array}$ & Ref. \\
\hline $\begin{array}{l}1986-08-15 \\
1998-10-15\end{array}$ & $\begin{array}{l}1 \\
1\end{array}$ & $\begin{array}{l}60000 \\
100000\end{array}$ & Ca II K & $\begin{array}{l}\text { 1.4-m Coudé Auxiliary Telescope (CAT)/La Silla } \\
\text { ESO Coudé Echelle Spectrometer (CES) }\end{array}$ & 1 \\
\hline $\begin{array}{l}1996-11-30 \\
1997-06-20 \\
2005-06-15 \\
\end{array}$ & $\begin{array}{l}1 \\
1 \\
1\end{array}$ & 1000000 & Ca II K & $\begin{array}{l}\text { 3.6-m Telescope/Anglo Australian Observatory } \\
\text { Ultra High Resolution Facility (UHRF) }\end{array}$ & $\begin{array}{l}2 \\
2 \\
3\end{array}$ \\
\hline $\begin{array}{l}1998-10-25 \\
1999-01-28\end{array}$ & $\begin{array}{l}1 \\
1\end{array}$ & 49000 & $380-590$ & $\begin{array}{l}\text { 4.2-m William Herschel Telescope/La Palma } \\
\text { Utrech Echelle Spectrograph (UES) }\end{array}$ & 4 \\
\hline $\begin{array}{l}2004-08-28 \\
2004-08-29 \\
2005-09-15\end{array}$ & $\begin{array}{l}1 \\
1 \\
1\end{array}$ & 240000 & Ca II K & $\begin{array}{l}\text { 2.7-m Harlan J. Smith Telescope/McDonald Observatory } \\
\text { Cross-Dispersed Echelle Spectrometer (CS21) }\end{array}$ & 3 \\
\hline $\begin{array}{l}2007-06-28 \\
2007-07-11 \\
2007-07-15 \\
2007-07-26 \\
2007-07-27 \\
\end{array}$ & $\begin{array}{c}14 \\
6 \\
14 \\
34 \\
6 \\
\end{array}$ & 60000 & $\begin{array}{l}360-450 \\
460-670\end{array}$ & $\begin{array}{l}\text { 8-m ESO-Very Large Telescope/Paranal } \\
\text { Ultraviolet and Visual Echelle Spectrograph (UVES) }\end{array}$ & $\begin{array}{l}\text { ESO } \\
\text { Archive }\end{array}$ \\
\hline $\begin{array}{l}2010-09-01 \\
2011-12-08\end{array}$ & $\begin{array}{l}3 \\
3\end{array}$ & & $336-810$ & $\begin{array}{l}\text { 10-m Keck I Telescope/Hawaii } \\
\text { High Resolution Echelle Spectrometer (HIRES) }\end{array}$ & $\begin{array}{c}\text { Keck } \\
\text { Archive }\end{array}$ \\
\hline $\begin{array}{l}2015-01-18 \\
2015-01-19 \\
2015-01-20 \\
2015-01-21\end{array}$ & $\begin{array}{l}1 \\
2 \\
1 \\
2\end{array}$ & 120000 & $378-691$ & $\begin{array}{l}\text { 3.6-m ESO Telescope/La Silla } \\
\text { High Accuracy Radial velocity Planet Searcher (HARPS) }\end{array}$ & $\begin{array}{l}\text { ESO } \\
\text { Archive }\end{array}$ \\
\hline
\end{tabular}

Notes. Dates of the specific nights of the campaigns are given in Table 3. " $N$ " is the number of spectra. Data from CARMENES will be used in a forthcoming paper.

References. (1) Lagrange-Henri et al. (1990), (2) Welsh et al. (1998), (3) Redfield et al. (2007), (4) EXPORT La Palma International Time campaigns, Mora et al. (2001).

\section{Spectroscopic observations}

In this section we describe the sets of high-resolution spectroscopic observations of HR 10 that have been used in this work to analyse the CS narrow absorption features superimposed on the photospheric lines, decompose their complex profiles, and measure the corresponding RVs of the components. We built a historical record covering the period 1986-2019 in order to complete what is to date the most comprehensive study of the CS variability of this object.

\subsection{Dedicated campaigns}

Table 2 (upper block) summarises the information of the campaigns coordinated by our team since September 2015 to observe HR 10, among other stars, looking for transient exocometary 


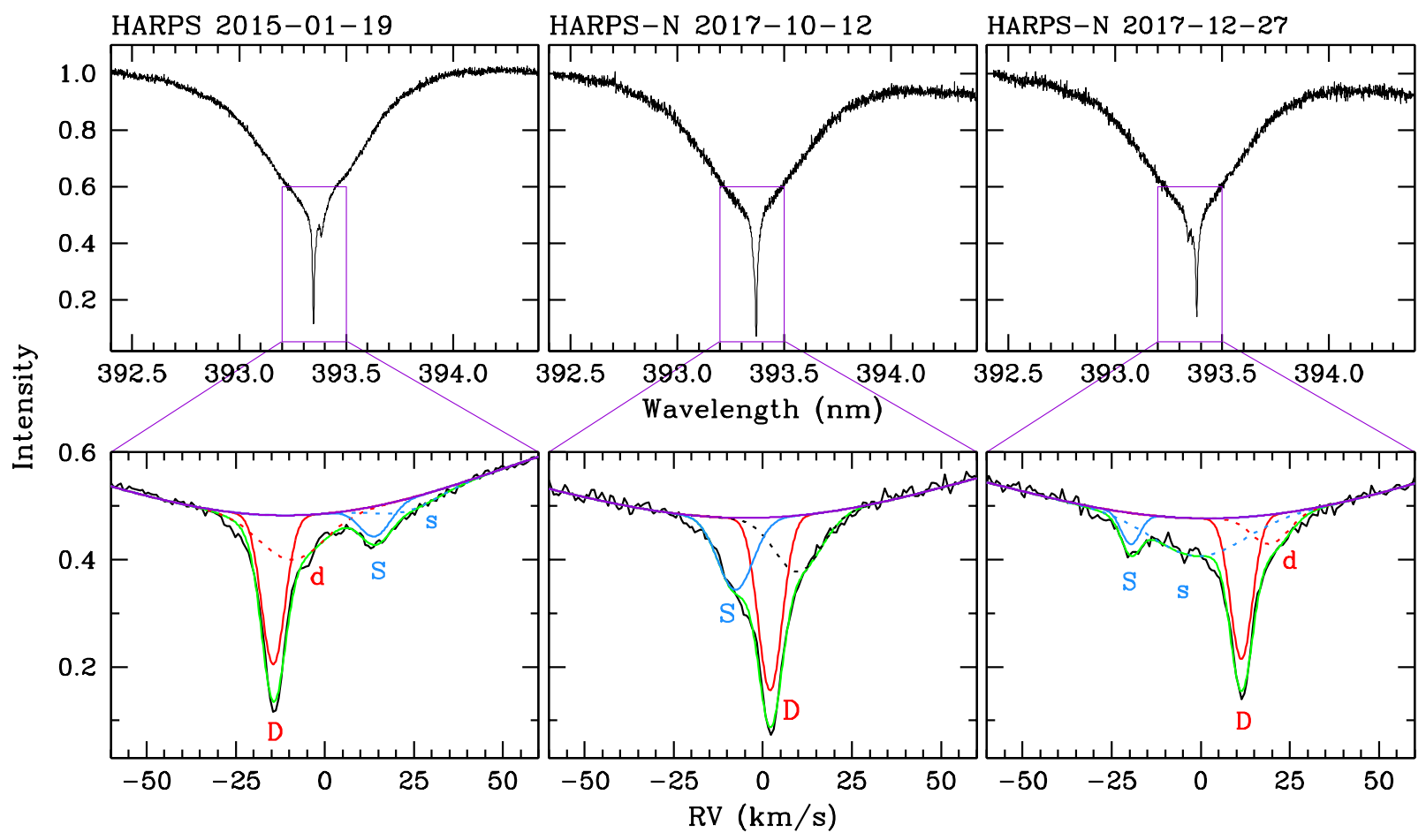

Fig. 2. Top: profiles of the broad photospheric Ca II K line of HR 10 of spectra obtained on 19 Jan 2015, 12 Oct 2017 and 27 Dec 2017 . Superimposed on the lower part of the lines, narrow absorption features are remarkably conspicuous. Bottom: narrow CS components corresponding to the spectra at the top, plotted in velocity space. The velocity $\mathrm{RV}=0 \mathrm{~km} \mathrm{~s}^{-1}$ corresponds to the rest wavelength of the Ca II $\mathrm{K}$ line. The letters D and $\mathrm{S}$ label the deep and shallow components of each CS absorption, respectively; these are present in all the spectra analysed in this work. Solid red and blue lines correspond to the Gaussian decomposition of those two main components, the bottom of the photospheric profile is plotted in purple and the whole fit in green. Dotted lines are the additional Gaussians d and s required to complete the decomposition of the absorption. See text for details.

events around MS stars, and campaigns targeted exclusively at HR 10. Signal-to-noise ratios (S/Ns) of the spectra at the bottom of the Ca II K line were always above 100. The spectra used in this work come from the corresponding reduction pipelines.

\subsection{Archival and published data}

In Table 2 (lower block) we give the relevant information for the observations obtained prior to September 2015, when we started our own monitoring campaigns. The data come from the literature, were provided by one of the authors (Prof. Seth Redfield), or were taken from observatory archives. Direct measurements on the reduced spectra, corrected for barycentric velocity, were done for the observations obtained with UES/WHT, CS21/ 2.7-m HJS, UVES/VLT, HIRES/Keck, and HARPS/3.6-m La Silla, and on the spectrum taken on 15 Jun 2005 with UHRF/ 3.6-m AAO. The spectra from Lagrange-Henri et al. (1990) were not available in readable format, and were therefore scanned from the paper itself and digitized. The data on radial velocities from the observations obtained on 30 Nov 1996 and 20 Jun 1997 were taken directly from the work by Welsh et al. (1998).

\section{The circumstellar absorption features}

\subsection{The Ca II K line}

Figure 2 (top) shows the profiles of the broad photospheric Ca II K line of spectra of HR 10 obtained on 19 Jan 2015, 12 Oct 2017, and 27 Dec 2017. Superimposed on the lower part of the lines, narrow absorption features are remarkably conspicuous. The insets delimited with purple boxes are blown up and plotted in velocity space at the bottom row; $\mathrm{RV}=0 \mathrm{~km} \mathrm{~s}^{-1}$ corresponds to the rest wavelength of the Ca II K line, namely, $393.366 \mathrm{~nm}$.
These three observations are representative of what we see in the whole set of spectra analysed in this work. The decomposition of the profiles in Gaussian components was carried out using the Emission Line Fitting (ELF) package of dipso, a spectrum analysis program developed by the Starlink Project (Howarth et al. 2004). The common feature of all the narrow absorptions is the presence of two main components: one deep and one shallow, hereafter plotted as red and blue solid lines, respectively, when the absorption is decomposed into Gaussian functions and labelled "D" and "S". The origin of these narrow absorption features is clearly CS, their variability being attributed in previous works to $\beta$ Pic-like phenomena (see Sect. 2), however the interpretation of their dynamical behaviour, as we see below, when analysing the RV series over a long time span turns out to be related to the binarity of HR 10 rather than to transient phenomena.

The profiles of the observations taken on 19 Jan 2015 and 27 Oct 2017 (left and right panels of Fig. 2) show two cases where the D and S components appear well separated, whereas the observation taken on 12 Oct 2017, plotted in the middle, shows a case where the separation in radial velocity between $D$ and $\mathrm{S}$ is small. The pattern observed during the $\sim 32 \mathrm{yr}$ of observations collected is that component $\mathrm{D}$ moves to the blue (red), as component $\mathrm{S}$ moves to the red (blue), crossing each other at certain times.

When the profile was such that the two main components appeared well separated, a four-Gaussian fit was needed to reproduce the whole narrow absorption. The two additional components, plotted as dotted red and blue lines, are placed to the red side of the main D and S components, respectively; these latter have been labelled as "d" and "s", to stress the fact that each one seems to be associated with D and S. When the profile 


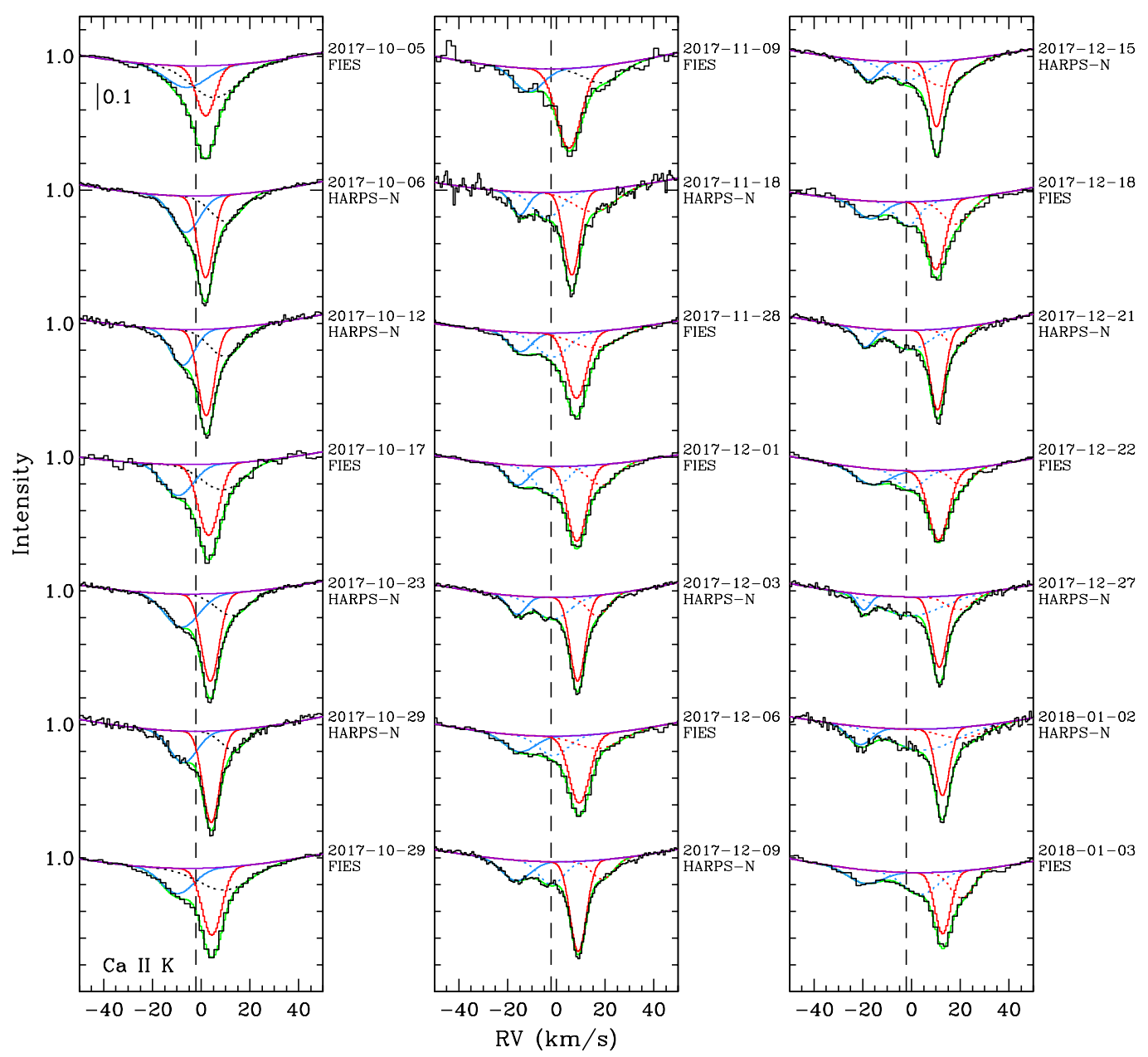

Fig. 3. Ca II K CS profile for 21 observations obtained with FIES/NOT and HARPS-N/TNG between 5 Oct 2017 and 3 Jan 2018 . The profiles have been decomposed with three or four Gaussians, plotting D (d) and S (s) as solid (dotted) red and blue lines. The pseudocontinuum tracing the bottom of the broad photospheric line is plotted in purple and the fit to the whole profile is plotted in green. For the three-Gaussian fit the additional component has been plotted as a dotted black line. The dashed line marks the RV of the system $-2.18 \pm 0.32 \mathrm{~km} \mathrm{~s}^{-1}$ (see Sect. 6.2 and Table 4).

showed an appearance similar to that plotted in the middle graph of Fig. 2, a three-Gaussian fit was accurate enough to reproduce the absorption. Whereas in the four-Gaussian fittings the $\mathrm{d}$ and s components, redshifted with respect to $\mathrm{D}$ and $\mathrm{S}$, seem to have a physical relationship with their corresponding main component, in the case of three-Gaussian fits, the parameters of the additional component probably embed the contribution of the two dotted components of the four-Gaussian decomposition. In Fig. 2 the pseudocontinuum tracing the bottom of the broad photospheric line is plotted in purple and the fit to the whole profile is plotted in green. For the three-Gaussian fit the additional component has been plotted as a dotted black line.

Figure 3 shows the results of a well-sampled monitoring carried out between 5 Oct 2017 and 3 Jan 2018 (21 spectra obtained with FIES/NOT and HARPS-N/TNG), where the gradual dynamical evolution of $\mathrm{D}$ and $\mathrm{d}$ moving to the red, and $\mathrm{S}$ and s moving to the blue, is very clear. The colour code is the same as in Fig. 2.

We quantitatively analyse the behaviour of all these components in Sects. 6.1 and 7.1.

\subsection{Circumstellar absorption features in other photospheric lines}

In this work we concentrate on the analysis of the Ca II $\mathrm{K}$ line for two main reasons: the first one is that this line is the most important and clear tracer of CS activity, as has been proved in $\beta$ Pic and other stars (see references in the Introduction); the second is that for some of the early spectroscopic observations, which have been crucial for the completion this study, only data for this particular line were available. However, HR 10 presents narrow CS absorption features superimposed on many other photospheric lines.

Figure 4 shows the CS components superimposed on 24 photospheric lines of the spectrum of HR 10. Since some of these components are fairly weak, in order to increase the $\mathrm{S} / \mathrm{N}$, five FIES/NOT spectra - those taken between 26 Dec 2018 and 8 Jan 2019, see Table 3 - were averaged. These were chosen because the separation in RVs of the Ca II K narrow absorption features is large, almost $30 \mathrm{~km} \mathrm{~s}^{-1}$, and therefore the CS components present in other lines would appear well separated. The photospheric profile of each line has been subtracted and the residual narrow absorption features have been scaled between intensities 0.5 and 1.0. The red and blue solid lines are the average $\mathrm{RVs}$ of the D and S components of the Ca II K absorption, and the dashed line marks the RV of the system. The Ca II K CS absorption is plotted with a thicker line type.

In most cases, the CS narrow absorption features follow the same pattern of intensity as in Ca II K, with the remarkable exception of the IR Ca II triplet, where a strong component is seen in the three lines at the velocity of the shallow component of the $\mathrm{Ca}$ II $\mathrm{K}$ absorption. Conversely, a weak absorption at the 

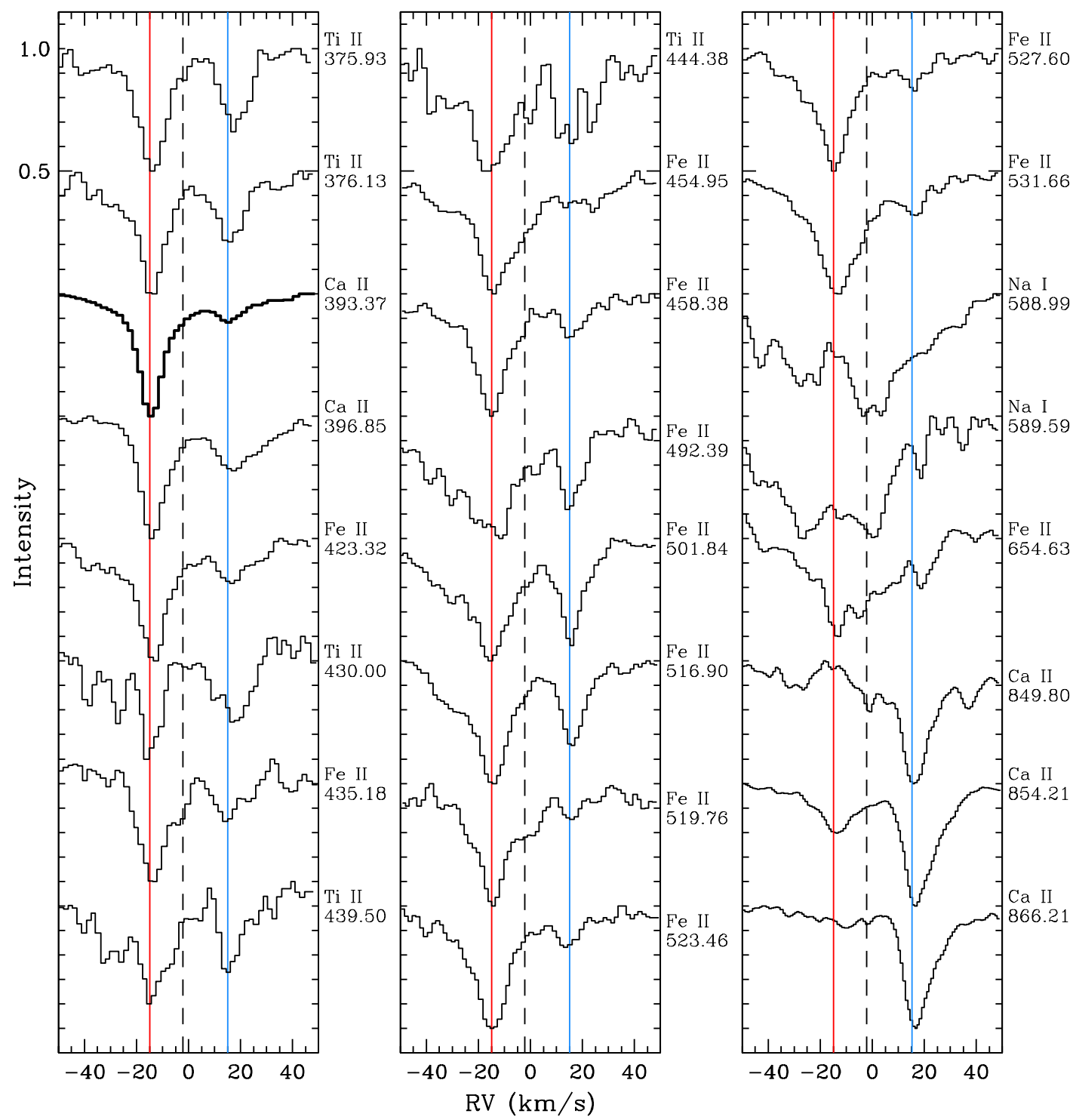

Fig. 4. Circumstellar components in 24 lines of the optical spectrum of HR 10. The photospheric profiles have been subtracted and, for the sake of clarity, the narrow absorption features have been scaled to the same size. The red and blue lines mark the radial velocities of the D (deep) and $\mathrm{S}$ (shallow) components, $-14.80 \pm 0.50+15.16 \pm 1.54 \mathrm{~km} \mathrm{~s}^{-1}$, respectively, of the Ca II K narrow absorption, which has been plotted with a thicker line. The dashed line marks the RV of the system, $-2.18 \pm 0.32 \mathrm{~km} \mathrm{~s}^{-1}$. The line identification with the wavelength in nm is included. See text for details.

velocity of the Ca II K strong component is clearly visible in the central and most intense line of the triplet $(854.21 \mathrm{~nm})$, but does not appear in the other two. On the other hand, the appearance of the absorption features at the $\mathrm{Na}$ I $\mathrm{D}$ doublet is complex due to the many telluric lines in that spectral range; these were removed using the package MOLECFIT ${ }^{1}$ (Smette et al. 2015; Kausch et al. 2015).

Figure 5 shows the narrow CS components superimposed on the photospheric $\mathrm{Ca}$ II $\mathrm{K}$ and $\mathrm{H}$ lines, the $\mathrm{Ti}$ II $375.93+$ $376.13 \mathrm{~nm}$ and the Fe II $423.32 \mathrm{~nm}$ lines; in the case of the Ti II doublet, the profiles of the two CS absorptions of the two lines, in velocity space, were merged and converted into a single profile. Colour codes and the meaning of the vertical lines are the same as in Figs. 2-4. It is remarkable that components $s$ and

\footnotetext{
1 http://www.eso.org/sci/software/pipelines/skytools/ molecfit
}

$\mathrm{d}$, which appear in the Gaussian decomposition of the Ca II K narrow absorptions, are also present in other species.

Figure 6 shows the bottom of the photospheric Balmer lines $\mathrm{H} \alpha$ and $\mathrm{H} \beta$ for two situations with the $\mathrm{D}$ and $\mathrm{S}$ components of $\mathrm{Ca}$ II $\mathrm{K}$ - plotted in purple as a reference - appear well separated (top) and blended (bottom). The profiles at the top correspond to the same spectra used in Fig. 4, whereas the profiles at the bottom are extracted from an average of the HARPS-N spectra obtained on 6 Oct 2017 and 24 Oct 2017. The vertical red and blue lines mark the RVs of components D and $\mathrm{S}$ and the dashed black line marks the RV of the system; three telluric features on the $\mathrm{H} \alpha$ profiles are marked by green crosses. It is very clear that the minima of the Balmer profiles are located at the RV of component D. The profiles at the top are clearly asymmetric. In the following section it becomes clear why this happens, the binarity being the origin of the variable shape and position of the bottom of these lines. 
Table 3. Radial velocities (RV) and velocity dispersions $(b)$ of the deep " $\mathrm{D}$ " and shallow "S" components of the CS Ca II K absorptions.

\begin{tabular}{|c|c|c|c|c|c|c|c|c|c|c|c|}
\hline \multirow{3}{*}{ Date } & \multirow{3}{*}{$N$} & \multirow{3}{*}{$\begin{array}{c}\text { BJD (TDB) } \\
\text { (days) }\end{array}$} & \multicolumn{8}{|c|}{ Component } & \multirow{3}{*}{ Instrumen } \\
\hline & & & \multicolumn{2}{|l|}{$\mathrm{D}$} & \multicolumn{2}{|l|}{$\mathrm{S}$} & \multicolumn{2}{|l|}{ d } & \multicolumn{2}{|l|}{$\mathrm{s}$} & \\
\hline & & & $\begin{array}{c}\mathrm{RV} \\
\left(\mathrm{km} \mathrm{s}^{-1}\right)\end{array}$ & $\begin{array}{c}b \\
\left(\mathrm{~km} \mathrm{~s}^{-1}\right) \\
\end{array}$ & $\begin{array}{c}\mathrm{RV} \\
\left(\mathrm{km} \mathrm{s}^{-1}\right)\end{array}$ & $\begin{array}{c}b \\
\left(\mathrm{~km} \mathrm{~s}^{-1}\right)\end{array}$ & $\begin{array}{c}\mathrm{RV} \\
\left(\mathrm{km} \mathrm{s}^{-1}\right)\end{array}$ & $\begin{array}{c}b \\
\left(\mathrm{~km} \mathrm{~s}^{-1}\right)\end{array}$ & $\begin{array}{c}\mathrm{RV} \\
\left(\mathrm{km} \mathrm{s}^{-1}\right)\end{array}$ & $\begin{array}{c}b \\
\left(\mathrm{~km} \mathrm{~s}^{-1}\right)\end{array}$ & \\
\hline $1986-08-15$ & 1 & 2446657.5000 & $-8.66 \pm 2.00$ & 10.1 & $+9.38 \pm 2.00$ & 9.0 & & & & & CES \\
\hline 1988-10-15 & 1 & 2447449.5000 & $-4.49 \pm 2.00$ & 9.8 & $+5.83 \pm 2.00$ & 10.3 & & & & & CES \\
\hline $1996-11-30^{(a)}$ & 1 & 2450417.5022 & $-10.40 \pm 2.00$ & 2.1 & $+7.40 \pm 2.00$ & 3.8 & & & & & UHRF \\
\hline $1997-06-20^{(a)}$ & 1 & 2450619.5011 & $+9.20 \pm 2.00$ & 3.0 & $-16.60 \pm 2.00$ & 2.6 & $+16.20 \pm 2.00$ & 4.5 & & & UHRF \\
\hline $1998-10-25$ & 1 & 2451112.4652 & $-16.03 \pm 2.00$ & 10.0 & $+10.00 \pm 2.00$ & 10.0 & $-6.51 \pm 3.00$ & 13.7 & $+20.01 \pm 3.00$ & 9.8 & UES \\
\hline $1999-01-28$ & 1 & 2451207.3363 & $-8.78 \pm 2.00$ & 10.0 & & & & & & & UES \\
\hline 2004-08-28 & 2 & 2453246.3741 & $-17.33 \pm 2.00$ & 8.5: & $+13.80 \pm 2.00$ & 7.9: & & & & & CS21 \\
\hline $\begin{array}{l}2004-08-29 \\
2004-10-19\end{array}$ & 1 & 2453297.7801 & $-14.77 \pm 2.00$ & & & & & & & & CS21 \\
\hline $2005-06-15$ & 1 & 2453537.3058 & $-2.10 \pm 2.00$ & 12.3: & & & & & & & UHRF \\
\hline 2005-09-15 & 1 & 2453628.8717 & $+9.32 \pm 2.00$ & 14.7 & $-17.38 \pm 2.00$ & 16.3: & & & $-4.43 \pm 2.00$ & 7.4 & CS21 \\
\hline $2007-06-28$ & 14 & 2454279.8221 & $-0.59 \pm 1.50$ & 7.8 & $-4.40 \pm 1.50$ & 7.0 & & & & & UVES \\
\hline 2007-07-11 & 6 & 2454292.8971 & $+0.31 \pm 1.50$ & 6.2 & $-6.04 \pm 1.50$ & 4.6 & & & & & UVES \\
\hline $2007-07-15$ & 14 & 2454296.9144 & $+0.91 \pm 1.50$ & 6.4 & $-6.07 \pm 1.50$ & 6.0 & & & & & UVES \\
\hline $2007-07-26$ & 34 & 2454307.7830 & $+1.27 \pm 1.50$ & 7.5 & $-8.96 \pm 1.50$ & 8.0 & & & & & UVES \\
\hline $2007-07-27$ & 6 & 2454308.7539 & $+2.18 \pm 1.50$ & 8.0 & $-7.82 \pm 1.50$ & 8.0 & & & & & UVES \\
\hline 2010-09-01 & 1 & 2455441.1252 & $-14.33 \pm 1.50$ & 10.5 & $+14.05 \pm 1.50$ & 9.8 & $-4.01 \pm 2.00$ & 8.4 & $+18.73 \pm 2.00$ & 10.4 & HIRES \\
\hline $2011-12-08$ & 1 & 2455903.7969 & $+15.17 \pm 1.50$ & 8.0 & $-24.10 \pm 1.50$ & 10.4 & $+23.67 \pm 2.00$ & 10.2 & $-4.97 \pm 2.00$ & 8.0 & HIRES \\
\hline 2015-01-18 & 1 & 2457041.4530 & $-14.41 \pm 1.50$ & 8.2 & $+14.05 \pm 1.50$ & 11.0 & $-4.00 \pm 2.00$ & 9.4 & $+24.10 \pm 2.00$ & 9.8 & HARPS \\
\hline 2015-01-19 & 2 & 2457042.4529 & $-14.45 \pm 1.50$ & 8.0 & $+14.15 \pm 1.50$ & 11.4 & $-6.24 \pm 2.00$ & 10.2 & $+23.30 \pm 2.00$ & 10.0 & HARPS \\
\hline $2015-01-20$ & 1 & 2457043.4527 & $-14.41 \pm 1.50$ & 8.8 & $+13.65 \pm 1.50$ & 11.0 & $-1.99 \pm 2.00$ & 11.0 & $+23.28 \pm 2.00$ & 9.7 & HARPS \\
\hline $2015-01-21$ & 2 & 2457044.4527 & $-14.58 \pm 1.50$ & 8.4 & $+14.22 \pm 1.50$ & 11.0 & $-3.99 \pm 2.00$ & 10.3 & $+23.03 \pm 2.00$ & 11.0 & HARPS \\
\hline $\begin{array}{l}2015-09-04 \\
2015-09-07\end{array}$ & 17 & 2457271.0769 & $+0.08 \pm 1.50$ & 9.4 & $-7.36 \pm 1.50$ & 13.1 & $+10.31 \pm 2.00$ & 15.8 & $+5.95 \pm 2.00$ & 11.1 & HERMES \\
\hline $\begin{array}{l}2015-10-22 \\
2015-10-23\end{array}$ & 3 & 2457318.1488 & $+5.41 \pm 1.50$ & 9.2 & $-14.88 \pm 1.50$ & 9.4 & $+14.78 \pm 2.00$ & 19.9: & $-3.22 \pm 2.00$ & 19.7: & FEROS \\
\hline $2015-12-20$ & 3 & 2457377.3295 & $+12.22 \pm 1.50$ & 10.5 & $-19.22 \pm 1.50$ & 11.9 & $+24.37 \pm 2.00$ & 10.2 & $-3.02 \pm 2.00$ & 13.0 & HERMES \\
\hline $2015-12-22$ & 2 & 2457379.3572 & $+13.03 \pm 1.50$ & 9.5 & $-22.19 \pm 1.50$ & 13.6 & $+25.85 \pm 2.00$ & 11.0 & $-4.11 \pm 2.00$ & 8.8 & HERMES \\
\hline $2015-12-23$ & 3 & 2457380.3501 & $+13.12 \pm 1.50$ & 9.6 & $-21.28 \pm 1.50$ & 10.0 & $+27.98 \pm 2.00$ & 11.0 & $-5.80 \pm 2.00$ & 14.0 & HERMES \\
\hline $2016-07-12$ & 1 & 2457581.7080 & $-0.63 \pm 1.50$ & 7.1 & $-5.95 \pm 1.50$ & 6.1 & & & & & HERMES \\
\hline 2017-10-05 & 1 & 2458031.5276 & $+1.84 \pm 1.50$ & 9.0 & $-6.05 \pm 1.50$ & 19.4 & & & & & FIES \\
\hline $2017-10-06$ & 1 & 2458032.5772 & $+1.80 \pm 1.50$ & 7.7 & $-6.36 \pm 1.50$ & 13.3 & & & & & HARPS-N \\
\hline $2017-10-12$ & 2 & 2458038.5469 & $+2.07 \pm 1.50$ & 7.9 & $-7.69 \pm 1.50$ & 11.5 & & & & & HARPS-N \\
\hline $2017-10-17$ & 1 & 2458043.5495 & $+3.00 \pm 1.50$ & 9.9 & $-9.46 \pm 1.50$ & 14.5 & & & & & FIES \\
\hline $\begin{array}{l}2017-10-23 \\
2017-10-24\end{array}$ & 2 & 2458050.4980 & $+3.18 \pm 1.50$ & 4.8 & $-9.12 \pm 1.50$ & 13.2 & $+15.99 \pm 2.00$ & 15.7 & $+4.14 \pm 2.00$ & 12.1 & HARPS-N \\
\hline $2017-10-29$ & 1 & 2458056.3919 & $+4.40 \pm 1.50$ & 9.5 & $-9.74 \pm 1.50$ & 15.5 & & & & & FIES \\
\hline $2017-10-29$ & 2 & 2458056.4748 & $+4.26 \pm 1.50$ & 5.4 & $-9.57 \pm 1.50$ & 17.0: & $+16.02 \pm 2.00$ & 20.5: & $+4.47 \pm 2.00$ & 16.1: & HARPS-N \\
\hline 2017-11-09 & 1 & 2458067.4778 & $+5.15 \pm 1.50$ & 11.9 & $-11.31 \pm 1.50$ & 13.9 & & & & & FIES \\
\hline 2017-11-18 & 2 & 2458076.3180 & $+6.38 \pm 1.50$ & 9.6 & $-15.05 \pm 1.50$ & 13.2 & $+20.01 \pm 2.00$ & 14.1 & $-5.61 \pm 2.00$ & 10.0 & HARPS-N \\
\hline $2017-11-28$ & 1 & 2458086.4462 & $+8.32 \pm 1.50$ & 10.4 & $-14.88 \pm 1.50$ & 11.4 & $+20.62 \pm 2.00$ & 10.0 & $-2.61 \pm 2.00$ & 12.1 & FIES \\
\hline 2017-12-01 & 1 & 2458089.4417 & $+8.48 \pm 1.50$ & 9.2 & $-15.91 \pm 1.50$ & 10.5 & $+18.97 \pm 2.00$ & 13.2 & $-2.84 \pm 2.00$ & 13.8 & FIES \\
\hline $2017-12-03$ & 2 & 2458091.3887 & $+8.71 \pm 1.50$ & 9.1 & $-15.84 \pm 1.50$ & 11.0 & $+20.45 \pm 2.00$ & 11.3 & $-3.97 \pm 2.00$ & 9.6 & HARPS-N \\
\hline $2017-12-06$ & 1 & 2458094.4387 & $+9.46 \pm 1.50$ & 10.9 & $-15.10 \pm 1.50$ & 12.2 & $+23.01 \pm 2.00$ & 10.0 & $-3.00 \pm 2.00$ & 10.9 & FIES \\
\hline 2017-12-09 & 2 & 2458097.3047 & $+8.99 \pm 1.50$ & 7.9 & $-16.55 \pm 1.50$ & 13.6 & $+19.03 \pm 2.00$ & 10.4 & $-1.95 \pm 2.00$ & 11.8 & HARPS-N \\
\hline $2017-12-15$ & 2 & 2458103.3163 & $+10.29 \pm 1.50$ & 9.5 & $-17.72 \pm 1.50$ & 10.8 & $+22.45 \pm 2.00$ & 11.0 & $-3.09 \pm 2.00$ & 11.5 & HARPS-N \\
\hline $2017-12-18$ & 1 & 2458106.3321 & $+10.02 \pm 1.50$ & 9.0 & $-17.03 \pm 1.50$ & 12.9 & $+18.61 \pm 2.00$ & 13.1 & $-1.62 \pm 2.00$ & 11.4 & FIES \\
\hline $2017-12-21$ & 2 & 2458109.3041 & $+10.67 \pm 1.50$ & 9.0 & $-19.01 \pm 1.50$ & 11.6 & $+21.65 \pm 2.00$ & 7.0 & $-4.00 \pm 2.00$ & 8.5 & HARPS-I \\
\hline $2017-12-22$ & 1 & 2458110.3674 & $+11.18 \pm 1.50$ & 9.8 & $-16.14 \pm 1.50$ & 15.8 & $+20.87 \pm 2.00$ & 9.8 & $-0.36 \pm 2.00$ & 12.3 & FIES \\
\hline $2017-12-27$ & 2 & 2458115.3078 & $+11.39 \pm 1.50$ & 7.2 & $-19.69 \pm 1.50$ & 11.2 & $+20.05 \pm 2.00$ & 12.2 & $-6.82 \pm 2.00$ & 7.0 & HARPS-N \\
\hline 2018-01-02 & 2 & 2458121.3055 & $+12.71 \pm 1.50$ & 7.3 & $-21.15 \pm 1.50$ & 12.1 & $+21.05 \pm 2.00$ & 10.1 & $-2.91 \pm 2.00$ & 9.0 & HARPS-N \\
\hline 2018-01-03 & 1 & 2458122.3461 & $+12.85 \pm 1.50$ & 9.6 & $-18.89 \pm 1.50$ & 10.5 & $+22.02 \pm 2.00$ & 10.0 & $-7.52 \pm 2.00$ & 10.0 & FIES \\
\hline $2018-11-23$ & 1 & 2458446.4436 & $-15.08 \pm 1.50$ & 10.0 & $+12.90 \pm 1.50$ & 12.5 & $-3.00 \pm 2.00$ & 10.1 & $+20.49 \pm 2.00$ & 10.0 & FIES \\
\hline 2018-11-26 & 1 & 2458449.3208 & $-14.90 \pm 1.50$ & 10.0 & $+14.01 \pm 1.50$ & 8.0 & $-5.88 \pm 2.00$ & 10.0 & $+24.75 \pm 2.00$ & 7.8 & FIES \\
\hline 2018-12-01 & 1 & 2458454.3734 & $-14.82 \pm 1.50$ & 9.8 & $+13.23 \pm 1.50$ & 14.5 & $-6.21 \pm 2.00$ & 10.2 & $+21.79 \pm 2.00$ & 8.7 & FIES \\
\hline 2018-12-07 & 1 & 2458460.3696 & $-15.12 \pm 2.00$ & 10.3: & $+12.52 \pm 2.00$ & 17.3: & & & $+21.66 \pm 3.00$ & 21.7: & FIES \\
\hline 2018-12-10 & 1 & 2458463.4205 & $-15.29 \pm 1.50$ & 10.0 & $+14.43 \pm 1.50$ & 8.0 & $-4.01 \pm 2.00$ & 10.0 & $+22.01 \pm 2.00$ & 10.0 & FIES \\
\hline $2018-12-22$ & 1 & 2458475.3701 & $-15.09 \pm 1.50$ & 10.7 & $+14.35 \pm 1.50$ & 8.1 & & & $+22.37 \pm 2.00$ & 10.6 & FIES \\
\hline $2018-12-24$ & 1 & 2458477.3687 & $-14.26 \pm 1.50$ & 10.7: & & & & & & & FIES \\
\hline $2018-12-26$ & 1 & 2458479.3677 & $-14.69 \pm 1.50$ & 10.1 & $+14.39 \pm 1.50$ & 8.0 & $-2.37 \pm 2.00$ & 10.9 & $+22.01 \pm 2.00$ & 9.0 & FIES \\
\hline $2018-12-27$ & 1 & 2458480.3449 & $-15.53 \pm 1.50$ & 10.0 & $+15.27 \pm 1.50$ & 9.3 & $-5.03 \pm 2.00$ & 10.5 & $+22.25 \pm 2.00$ & 8.9 & FIES \\
\hline 2019-01-02 & 1 & 2458486.3505 & $-14.80 \pm 1.50$ & 9.6 & $+16.06 \pm 1.50$ & 8.2 & $-5.28 \pm 2.00$ & 10.0 & $+24.16 \pm 2.00$ & 8.0 & FIES \\
\hline 2019-01-06 & 1 & 2458490.3547 & $-14.84 \pm 1.50$ & 9.8 & $+17.04 \pm 1.50$ & 8.5 & $-5.48 \pm 2.00$ & 12.4 & $+22.01 \pm 2.00$ & 8.1 & FIES \\
\hline 2019-01-08 & 1 & 2458492.3427 & $-14.15 \pm 1.50$ & 9.6 & $+13.04 \pm 1.50$ & 7.9 & $-4.20 \pm 2.00$ & 9.9 & $+22.95 \pm 2.00$ & 8.0 & FIES \\
\hline
\end{tabular}

Notes. For those cases when a four-Gaussian fit was feasible, the parameters of the weak "d" and "s" components that appear redshifted with respect to D and S, respectively, are also given. " $N$ " is the number of spectra. ${ }^{(a)}$ Radial velocities and velocity dispersions for these two dates have been taken from Welsh et al. (1998), since the observations were not available for direct measurements following the same method used in this work. (:) Noisy spectra. 


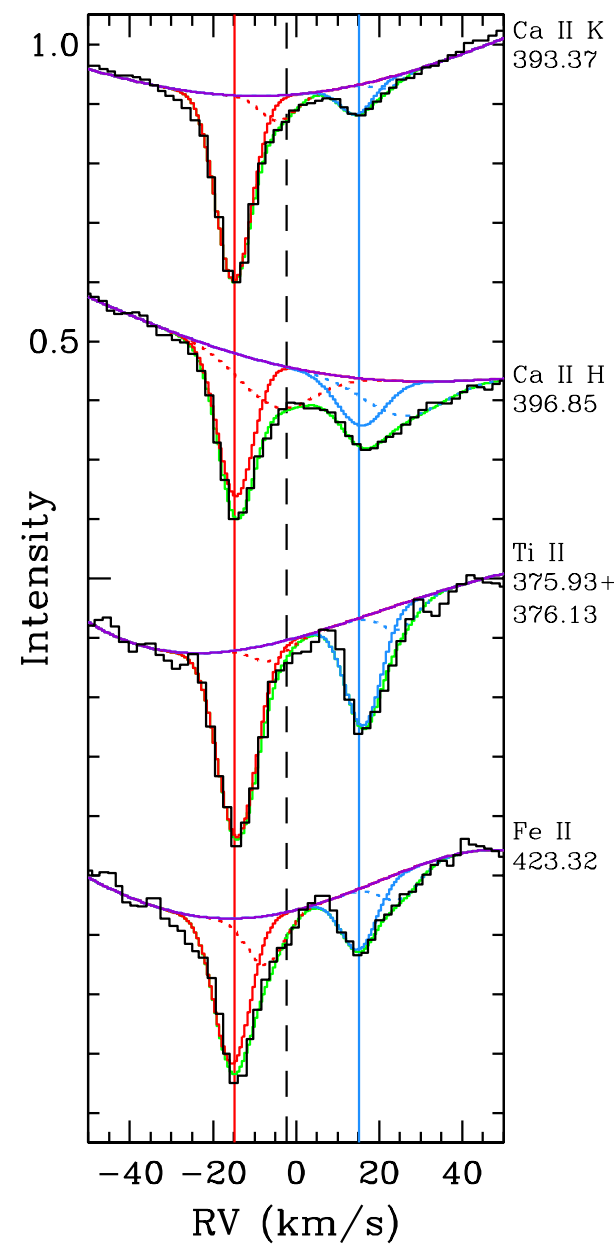

Fig. 5. Circumstellar components of the narrow absorption features superimposed to the photospheric Ca II $\mathrm{K}$ and $\mathrm{H}$ lines, the Ti II doublet around $376.0 \mathrm{~nm}$, and the Fe II line at $423.32 \mathrm{~nm}$, for the same set of observations as in Fig. 4; the vertical solid red and blue lines and the dashed black line mark the same radial velocities as in that figure. The Gaussian decomposition clearly shows the presence of the S, D (solid red and blue lines), $\mathrm{s}$, and d (dotted red and blue lines) components. The line identification with the wavelength in $\mathrm{nm}$ is included. See text for details.

We defer to a forthcoming paper a more detailed analysis of the CS absorption features in lines other than $\mathrm{Ca}$ II K, and also of the Balmer lines.

\section{Results}

\subsection{Radial velocities and velocity dispersions}

Table 3 shows the RVs and velocity dispersions, $b$, of the deep, D, and shallow, S, components of the narrow CS absorption features superimposed on the bottom of the photospheric $\mathrm{Ca}$ II $\mathrm{K}$ line, measured for all spectra. In those cases where a four-Gaussian fit was feasible, the same results for the $\mathrm{d}$ and $\mathrm{s}$ components are listed. The RVs correspond to the minimum of the main Gaussian components of the absorption (see Sect. 5) and the dispersion velocities are the full widths of the Gaussian profiles. All the values correspond to spectra obtained on individual nights; only in three cases were the measurements carried out over averaged spectra obtained on consecutive nights, namely 28-29 Aug 2004 (CS21), 4 to 7 Sep 2015 (HERMES), and 23-24 Oct 2017 (HARPS-N), to increase the S/N of the profile.

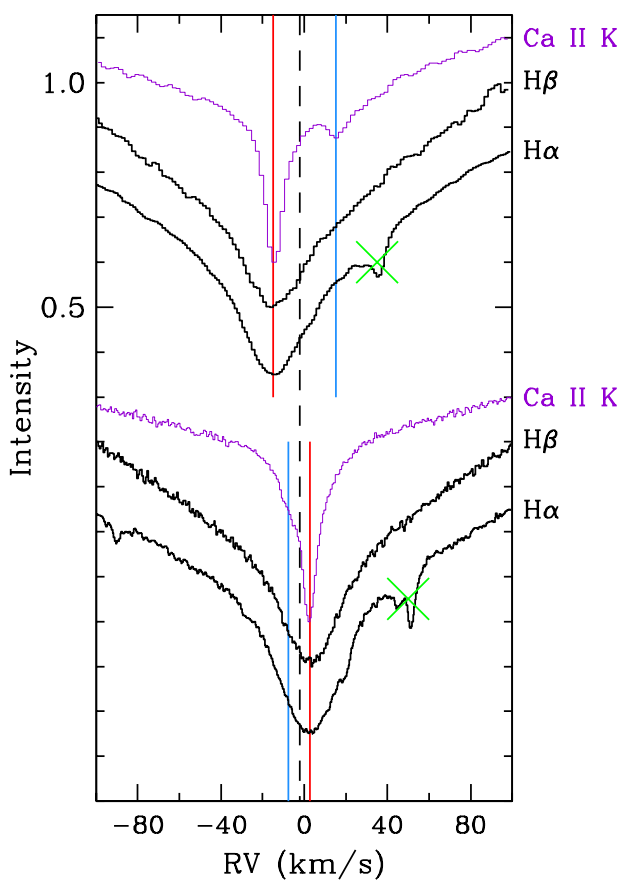

Fig. 6. Bottom of the Balmer lines $\mathrm{H} \alpha$ and $\mathrm{H} \beta$ for observations corresponding to a configuration where the difference in RVs is large (top) and small (bottom). The red and blue lines mark the radial velocities of the D and $\mathrm{S}$ components, measured on the Ca II K narrow absorption features, plotted in purple as references. Those RV are $-14.80 \pm$ 0.50 (D),$+15.16 \pm 1.54 \mathrm{~km} \mathrm{~s}^{-1}$ (S) (top), and $+2.75 \pm 0.73$ (D), $-7.72 \pm$ $1.38(\mathrm{~S}) \mathrm{km} \mathrm{s}^{-1}$ (bottom). The dashed line marks the RV of the system, $-2.18 \pm 0.32 \mathrm{~km} \mathrm{~s}^{-1}$. The two green crosses mark the position of telluric lines.

Uncertainties were assigned in a very conservative way taking into account those provided by the ELF package after the Gaussian decomposition of the profiles, and the effect of potential offsets between the wavelength calibrations provided by the pipelines of the different instruments. The quantitative effects of these offsets can be estimated by measuring the position of the telluric lines in spectra from the different spectrographs; differences in velocities were found to be less than $\sim 0.2 \mathrm{~km} \mathrm{~s}^{-1}$.

Figure 7 shows a plot of the whole set of RVs for the D (red) and $\mathrm{S}$ (blue) components given in Table 3 with the corresponding error bars. With the only purpose of guiding the eye, we have included in the graph two sinusoidal curves, plotted in pale red and blue, without any quantitative physical meaning, to show that the behaviour of the RV of each component seems to follow a periodic pattern over decades. The most plausible scenario is that each component, $\mathrm{D}$ and $\mathrm{S}$, traces the orbit of each individual star in the binary. In the following sections we prove, by means of robust calculations of a spectrometric binary solution (Sect. 6.2) and an orbital solution (Sect. 6.3), that this hypothesis is consistent.

\subsection{Spectrometric binary solution}

In this section we derive the spectrometric solution for the binary under the assumption mentioned in the last paragraph. We assign component D to HR 10-A, and component S to HR 10-B, the reason for this being, as we show in the following, that component D originates in the more massive of the two stars (component A) as it displays a smaller amplitude in the RV variation than component S. 


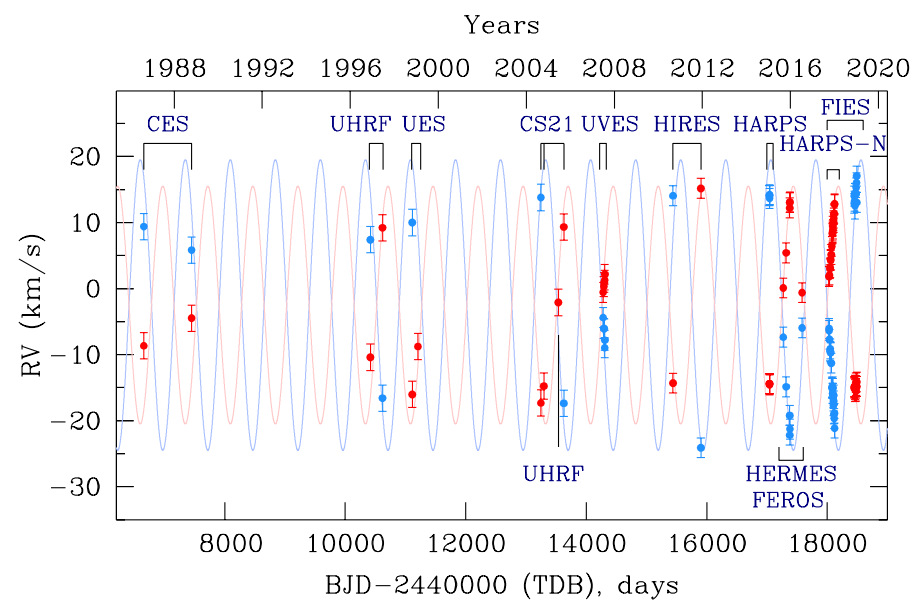

Fig. 7. Radial velocities of the components D (red) and S (blue) of the CS Ca II K narrow absorption features for all the observations. The sinusoidal curves plotted as pale red and blue have the only purpose to show, in a qualitative way, that the behaviour of the RVs shows a periodic pattern during the $\sim 32-y r$ interval covered by the observations. The exact treatment of the data is explained in Sect. 6.2. The labels indicate the instruments with which the observations were obtained (see Sect. 4).
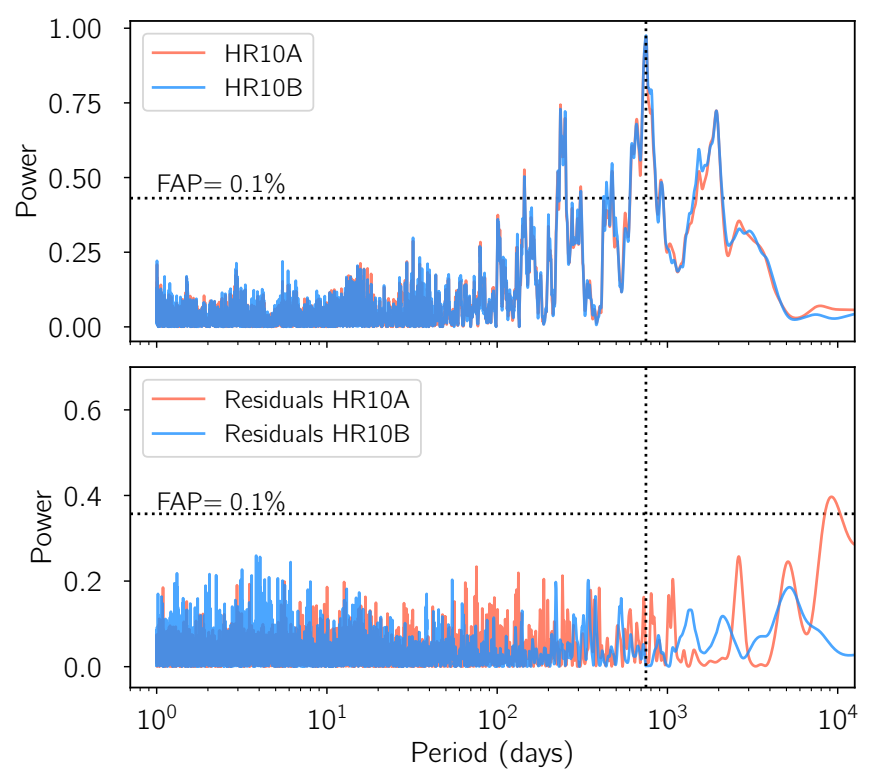

Fig. 8. Top: periodogram corresponding to the RV measurements for each of the two components of the binary system in HR 10 . The $0.1 \%$ FAP is shown as a horizontal dotted line and the final orbital period is displayed as a vertical dotted line. Bottom: periodogram of the residuals of the RV modelling.

We first computed the Lomb-Scargle periodogram of the measured RVs for each star using the astroml package (Ivezić et al. 2014) based on the methodology described in Zechmeister \& Kürster (2009). This periodogram is shown in the upper panel of Fig. 8. The result clearly shows a common peak above the $0.1 \%$ false-alarm probability (FAP) corresponding to a periodicity of $\approx 747$ days, indicating that both are gravitationally bound. Although the periodogram shows other peaks above the $0.1 \%$ FAP level, we attribute their origin to the different offsets between the many different instruments used, which are not taken into account in this periodogram computation but are in the modelling of the RV.
Table 4. Median and $68.7 \%$ confidence intervals for the RV parameters analysed in Sect. 6.2.

\begin{tabular}{lll}
\hline \hline Parameter & Prior & Posterior \\
\hline$V_{\text {sys }}\left(\mathrm{km} \mathrm{s}^{-1}\right)$ & $\mathcal{U}(-30.0,30.0)$ & $-2.18_{-0.31}^{+0.34}$ \\
$P_{\text {orb }}($ days $)$ & $\mathcal{G}(747.0,20.0)$ & $747.60_{-0.70}^{+0.61}$ \\
$T_{\mathrm{c}, \mathrm{A}}(\mathrm{BJD}-2400000)$ & $\mathcal{G}(57562.4,20.0)$ & $57563.5_{-7.2}^{+8.0}$ \\
$K_{\mathrm{A}}\left(\mathrm{km} \mathrm{s}^{-1}\right)$ & $\mathcal{U}(0.0,40.0)$ & $16.45_{-0.79}^{+0.76}$ \\
$e_{\mathrm{A}}$ & $\mathcal{U}(0.0,0.9)$ & $0.254_{-0.039}^{+0.036}$ \\
$\omega_{\mathrm{A}}\left(^{\circ}\right)$ & $\mathcal{U}(-180.0,180.0)$ & $38.0_{-4.0}^{+4.0}$ \\
$T_{\mathrm{c}, \mathrm{B}}(\mathrm{BJD}-2400000)$ & $\mathcal{U}(57562.0,58309.0)$ & $58022.3_{-8.6}^{+6.4}$ \\
$K_{\mathrm{B}}\left(\mathrm{km} \mathrm{s}^{-1}\right)$ & $\mathcal{U}(0.0,40.0)$ & $20.35_{-0.72}^{+0.69}$ \\
$e_{\mathrm{B}}$ & $\mathcal{U}(0.0,0.9)$ & $0.209_{-0.026}^{+0.030}$ \\
\hline
\end{tabular}

Notes. Prior distributions are also given. $\mathcal{U}(a, b)$ stands for uniform prior between $a$ and $b$, while $\mathcal{G}(\mu, \sigma)$ represents a Gaussian prior with mean $\mu$ and standard deviation $\sigma$.

We analyse the RV sets obtained from each component, D and $\mathrm{S}$ - corresponding to stars $\mathrm{A}$ and $\mathrm{B}$ - by modelling the whole system. The model includes two Keplerian orbits described by

$V_{\text {rad A }}=V_{\text {sys }}+K_{\mathrm{A}}\left[\cos \left(\nu_{\mathrm{A}}+\omega_{\mathrm{A}}\right)+e_{\mathrm{A}} \cos \left(\omega_{\mathrm{A}}\right)\right]$,
$V_{\mathrm{radB}}=V_{\mathrm{sys}}+K_{\mathrm{B}}\left[\cos \left(\nu_{\mathrm{B}}+\omega_{\mathrm{A}}+\pi\right)+e_{\mathrm{B}} \cos \left(\omega_{\mathrm{A}}+\pi\right)\right]$,

where $V_{\text {sys }}$ is the systemic velocity of the binary system, $\omega$ is the argument of the periastron of star $\mathrm{A}, e_{\mathrm{A}}$ and $e_{\mathrm{B}}$ are the eccentricities of the orbits of stars $\mathrm{A}$ and $\mathrm{B}$, respectively, and $v_{\mathrm{A}}$ and $v_{\mathrm{B}}$ are the true anomalies of each star, which implicitly include the times of conjunction $T_{\mathrm{c}, \mathrm{A}}$ and $T_{\mathrm{c}, \mathrm{B}}$. We used uninformative priors for all the parameters except for the orbital period, $P_{\text {orb }}$, and the conjunction passage. For $P_{\text {orb }}$, we used a normal distribution around the highest peak of the RV periodogram. The prior for the conjunction passage was set after a first exploration with an uninformative prior. The boundaries of the priors for all parameters are specified in Table 4 . Additionally, we included $N_{\text {inst }}-1$ additional parameters to account for RV offsets between the different instruments, with $N_{\text {inst }}$ being the number of instruments used. We set uninformative priors for these parameters between -1 and $+1 \mathrm{~km} \mathrm{~s}^{-1}$. In total, 19 parameters were explored.

In order to sample the posterior probability distribution of each of those parameters, we used the implementation of Goodman \& Weare's affine invariant Markov chain Monte Carlo (MCMC) ensemble sampler emcee developed by ForemanMackey et al. (2013). We used 50 walkers and 5000 steps per walker. This turned out to be enough due to the quick convergence of the chains. In order to compute the final posterior distributions, we discarded the first half of the chains and combined the second half to build the marginalised posteriors, finally composed of $1.25 \times 10^{5}$ steps. The median and $68.7 \%$ confidence intervals for each parameter are shown in Table 4 . The results of the final fitting are displayed in Fig. 9 showing the whole time span and Fig. 10 showing the phase-folded RV curves; this figure also includes the RVs of components $d$ and $s$, the behaviour of which is described in Sect. 7.1.

\subsection{Orbital solution}

The four PIONIER astrometric positions of the two stellar components of the binary system were analysed following the 


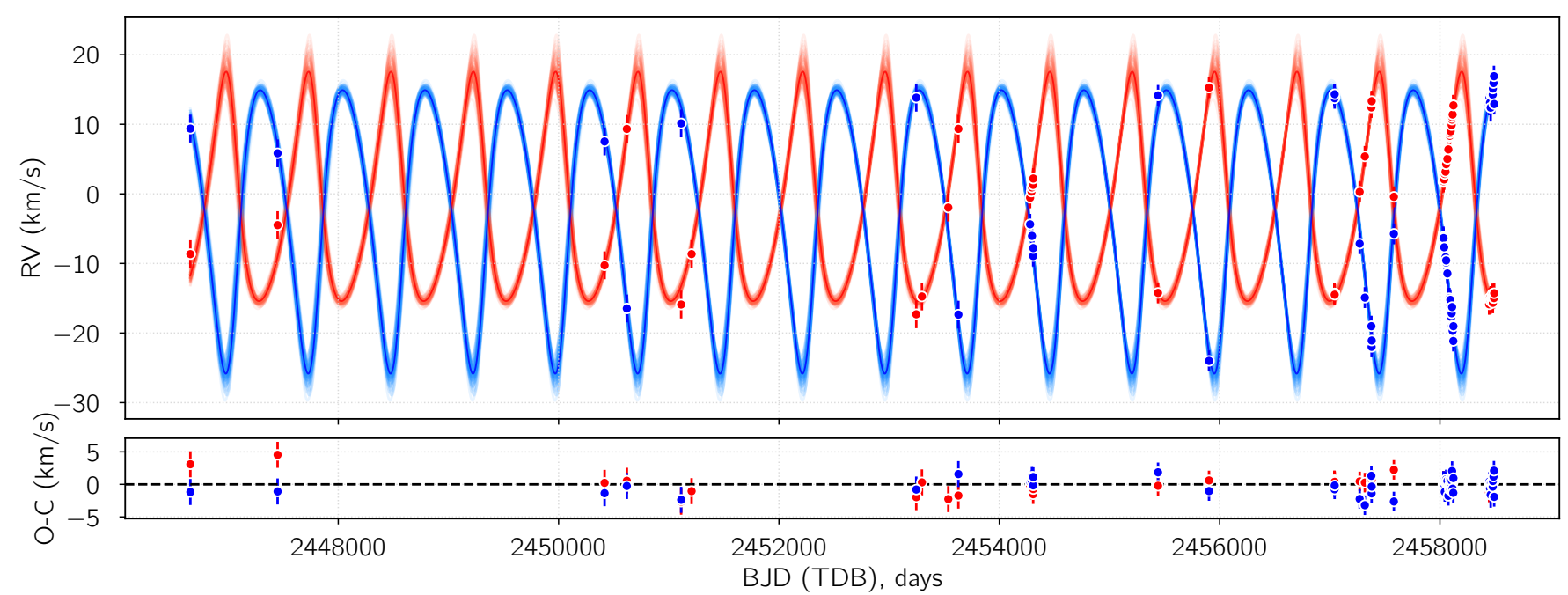

Fig. 9. Radial velocity measurements of the two stars along the $\sim 32 \mathrm{yr}$ of observations. Red and blue symbols and lines correspond to components $\mathrm{D}$ and S from the Gaussian fittings of the narrow Ca II K absorptions, which in turn correspond to stars A and B, respectively. Upper panel: model corresponding to the median values of the posterior distributions of the parameters as thick lines and 100 random samples drawn from the final MCMC chain; lower panel: residuals.

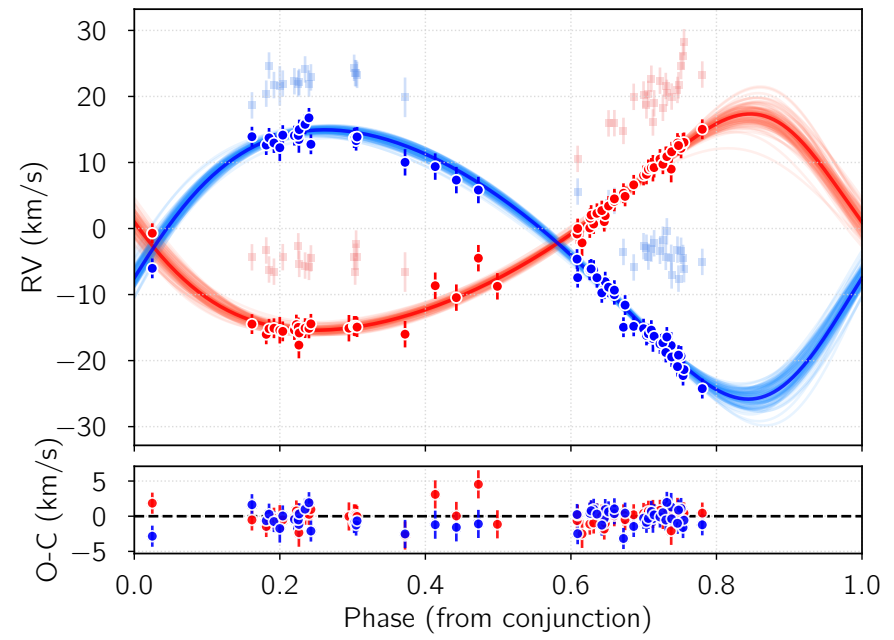

Fig. 10. Phase-folded RV curves of the two stellar components of HR 10. Red and blue circles and lines follow the same colour code as in Fig. 9. Pale red and blue squares correspond to the RVs of components $\mathrm{d}$ and $\mathrm{s}$; further details of their behaviour are given in Sect. 7.1.

equations of motion described in Mede \& Brandt (2017, see their Eqs. (7)-(15)). Given the low number of epochs, we assumed Gaussian priors on the orbital period, $P_{\text {orb }}$, and the eccentricities of the two orbits, $e_{\mathrm{A}}$ and $e_{\mathrm{B}}$, as obtained from the RV analysis. For the rest of the parameters, namely the argument of the periastron of star A, $\omega_{\mathrm{A}}$, the longitude of the ascending node of star A, $\Omega_{\mathrm{A}}$, orbital inclination, $i$, time of periapsis $T_{0}$, and the total semi-major axis, $a_{\mathrm{tot}}=a_{\mathrm{A}}+a_{\mathrm{B}}$, we used uninformative priors as stated in Table 5. The posterior distribution of these parameters was explored using again the emcee code. We used 20 walkers and 10000 steps per walker to widely explore the parameter space and then ran a second exploration in a smaller parameter space centred on the maximum likelihood set of parameters as obtained from the first phase. In this second run, we used 20 walkers and 5000 steps. The first $20 \%$ of the steps for each walker were removed and the posterior distributions were built from a total of 80000 steps. All chains converged nicely into the values presented in Table 5, including the median and $68.7 \%$
Table 5. Median and $68.7 \%$ confidence intervals for the orbital parameters analysed in Sect. 6.3.

\begin{tabular}{lll}
\hline \hline Parameter & Prior & Posterior \\
\hline$P_{\text {orb }}($ days $)$ & $\mathcal{G}(747.58441074,0.67)$ & $747.36_{-0.64}^{+0.67}$ \\
$T_{0}(\mathrm{BJD}-2400000)$ & $\mathcal{U}(57200.0,57600.0)$ & $57453_{-44}^{+22}$ \\
$a_{\text {tot }}(\mathrm{mas})$ & $\mathcal{U}(10.0,50.0)$ & $10.58_{-0.37}^{+0.60}$ \\
$\Omega_{\mathrm{A}}\left(^{\circ}\right)$ & $\mathcal{U}(0,360)$ & $137.65_{-0.84}^{+0.83}$ \\
$\omega_{\mathrm{A}}\left(^{\circ}\right)$ & $\mathcal{U}(-180.0,180.0)$ & $17_{-22}^{+11}$ \\
$i\left(^{\circ}\right)$ & $\mathcal{U}(90.0,100.0)$ & $93.34_{-0.63}^{+0.60}$ \\
$e_{\mathrm{A}}$ & $\mathcal{G}(0.24,0.03)$ & $0.242_{-0.012}^{+0.017}$ \\
$e_{\mathrm{B}}$ & $\mathcal{G}(0.207,0.03)$ & $0.208_{-0.011}^{+0.015}$ \\
\hline
\end{tabular}

Notes. Prior distributions are also displayed. $\mathcal{U}(a, b)$ stands for uniform prior between $a$ and $b$, while $\mathcal{G}(\mu, \sigma)$ represents a Gaussian prior with mean $\mu$ and standard deviation $\sigma$.

confidence intervals. As an example, the result for the argument of the periastron obtained from this analysis is in perfect agreement (within $2 \sigma$ ) with the result from the RV analysis (see Table 4). Figure 11 shows the positions of the stars in the plane of the sky according to the four PIONIER/VLTI observations, and the solutions for the projected orbits of HR 10-A (red) and HR 10-B (blue) around the centre of mass.

\subsection{Stellar parameters}

In order to compute a consistent set of stellar parameters, the observed photometry, spectra, and some of the quantities extracted from the binary solution, must be matched by the combination of the individual synthetic photometry and spectra of each component. The following inputs have been used:

1. High-resolution optical spectra.

2. Low-resolution ultraviolet spectra: HR 10 was observed with the International Ultraviolet Explorer (IUE, Kondo \& Wamsteker 1987). A merged spectrum covering the interval 


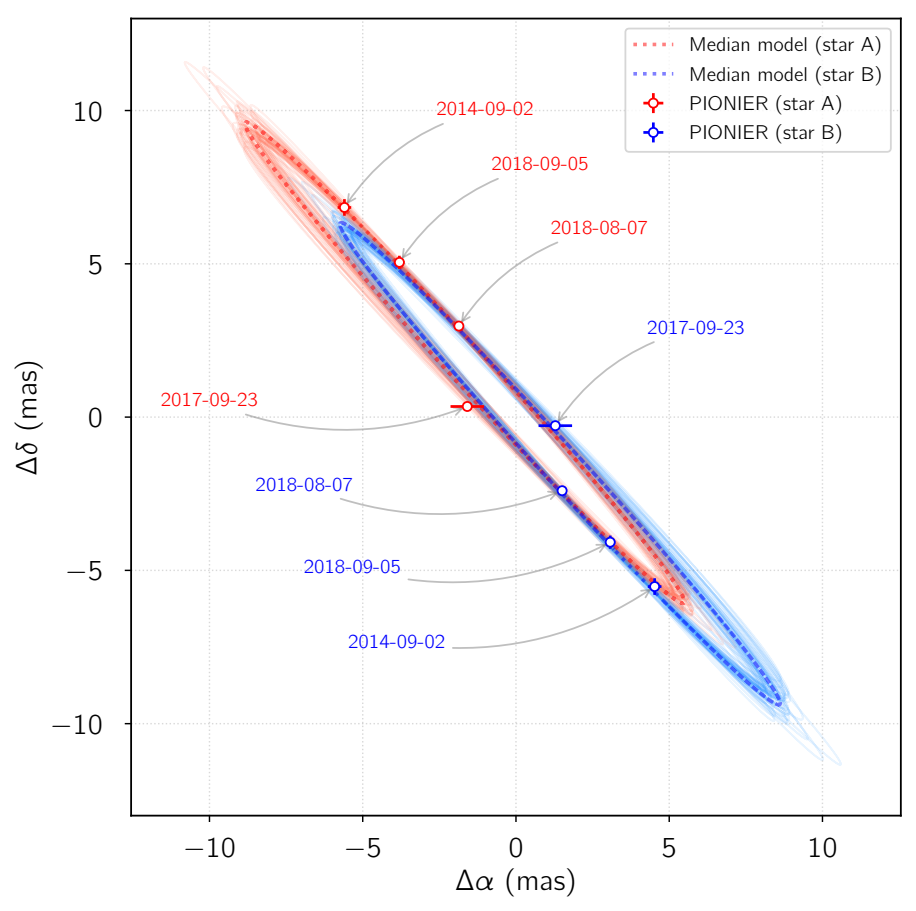

Fig. 11. PIONIER/VLTI astrometric positions for the two components of the HR10 resolved binary system (circle symbols include error bars). The date for each observation is specified. A sample of 100 orbital solutions obtained from the posterior distributions derived in Sect 6.3 (see Table 5), are represented by the colour-coded thin lines. The median models are represented by thick dashed lines.

115.0-332.0 nm was built from spectra SWP38943 and LWP18015 extracted from the IUE Newly Extracted Spectra (INES) database ${ }^{2}$.

3. Photometry: optical and NIR photometry of HR 10 collected from several sources is given in Table 6. Johnson $B V$ are from the SIMBAD database, and PAN-STARSS DR1 grizy and 2MASS $J H K_{\mathrm{s}}$ are taken from VizieR catalogues II/349/ps1 (Chambers et al. 2016) and II/246 (Cutri et al. 2003), respectively. The calibration from magnitudes into fluxes was carried out using the zero points from Bessell (1979) and Cohen et al. (2003) for $B V$ and $J H K_{\mathrm{s}}$, respectively; grizy are $\mathrm{AB}$ magnitudes and a value of $F_{0}=3631.0 \mathrm{Jy}$ has been adopted. The effective wavelength, $\lambda_{\text {eff }}$, for each filter is provided. An uncertainty of 0.02 mag was assigned to the optical magnitudes, since no value is given in the corresponding catalogues.

4. Relative brightness of the stars constrained by the contrast in $H$-band $\left(F_{H}(\mathrm{~B}) / F_{H}(\mathrm{~A}) \simeq 0.32\right)$ from the PIONIER observations (see Table 1).

5. Masses derived from the binary solution: provided the inclination of the system is known, the individual masses of the components can be estimated with the following expression:

$$
M_{\mathrm{A}(\mathrm{B})} \sin ^{3} i=\frac{P_{\text {orb }}}{2 \pi G}\left(1-e_{\mathrm{A}(\mathrm{B})}^{2}\right)^{3 / 2}\left(K_{\mathrm{A}}+K_{\mathrm{B}}\right)^{2} K_{\mathrm{B}(\mathrm{A})} .
$$

Using the data in Tables 4 and 5 we obtain $M_{\mathrm{A}}=1.94 \pm 0.15$, and $M_{\mathrm{B}}=1.62 \pm 0.13 M_{\odot}$.

The starting point of the estimation of the stellar parameters is a one-model fitting of the optical high-resolution spectrum, using Kurucz synthetic models; the method is described in Rebollido et al. (2018); the programmes SYNTHE and ATLAS (Kurucz

\footnotetext{
2 http://sdc.cab.inta-csic.es/ines/
}

Table 6. HR 10: optical and NIR photometry.

\begin{tabular}{lrl}
\hline \hline Magnitude & $\lambda_{\text {eff }}(\mathrm{nm})$ & \multicolumn{1}{c}{ Filter } \\
\hline 6.33 & 440.0 & $B$ Johnson/Bessell \\
6.25 & 477.6 & PAN-STARRS/PS1.g \\
6.230 & 550.0 & $V$ Johnson/Bessell \\
6.328 & 612.9 & PAN-STARRS/PS1.r \\
6.434 & 748.5 & PAN-STARRS/PS1.i \\
6.507 & 865.8 & PAN-STARRS/PS1.z \\
6.539 & 960.3 & PAN-STARRS/PS1.y \\
$5.858 \pm 0.019$ & 1235.0 & 2MASS $J$ \\
$5.831 \pm 0.033$ & 1662.0 & 2MASS $H$ \\
$5.747 \pm 0.027$ & 2159.0 & 2MASS $K_{\mathrm{s}}$ \\
\hline
\end{tabular}

1993) fed with models describing the stratification of the stellar atmospheres (Castelli \& Kurucz 2003) have been used for the spectral synthesis. Since the computation of the effective temperature hinges on the match of the depth of the photospheric $\mathrm{Ca}$ II $\mathrm{K}$ profile, we derived a model that slightly underestimates that depth, measured on a spline fitted to the line profile, avoiding the CS components. The reason for this is that the final fit will be composed of two models, therefore the addition of the synthetic $\mathrm{Ca}$ II K profiles of both stars should match the observed profile. The spectral fit was made on observations obtained with the stars almost in conjunction, to avoid large shifts between the spectral lines of both components. After some iterations, the result is $T_{\text {eff }}=9000 \pm 100 \mathrm{~K}, \log g_{*}=3.8 \pm 0.1$; the model was broadened with $v \sin i=294 \mathrm{~km} \mathrm{~s}^{-1}$ (Mora et al. 2001). We make the initial hypothesis that the parameters of star A are close to these ones; this hypothesis is tested at the end of the fitting process.

Since the contrast in $H$-band between the two components is 0.32 , it is trivial to deduce that $\Delta H(\mathrm{~A}-\mathrm{B})=-1.24$. Magnitudes $B_{\mathrm{A}}, V_{\mathrm{A}}, J_{\mathrm{A}}, H_{\mathrm{A}}$ and $K_{\mathrm{s}, \mathrm{A}}$ for star A, with $T_{\mathrm{eff}}(\mathrm{A})=9000 \mathrm{~K}$ and $\log g_{*}(\mathrm{~A})=3.8$ have been computed. In order to estimate the parameters of star $\mathrm{B}$, a grid of magnitudes $B_{\mathrm{B}}, V_{\mathrm{B}}, J_{\mathrm{B}}, H_{\mathrm{B}}$ and $K_{\mathrm{s}, \mathrm{B}}$ was created, covering a range of $T_{\text {eff }}$ from 7500 to $9000 \mathrm{~K}$ (step $50 \mathrm{~K}$ ), and $\log g_{*}$ from 3.8 to 4.3 (step $0.1 \mathrm{dex}$ ). The empirical colour-temperature calibration by Worthey \& Lee (2011) and the code mash3.f provided by these authors were used ${ }^{3}$. The sets of magnitudes were computed from the corresponding colours keeping $\Delta H(\mathrm{~A}-\mathrm{B})=-1.24$.

Combined colours $B-V, V-H, J-H$ and $J-K_{\mathrm{s}}$ were computed using the magnitudes of both stars and reddened with values of $E(B-V)$ between 0.0 and 0.2 ; the coefficients $A_{\lambda} / A_{V}$ were taken from Wang \& Chen (2019, Table 3); independent extinctions were allowed for each star in order to take into account potential differences caused by the individual CS envelopes. These synthetic colours are compared with the observed ones, this process yielding a subset of solutions [9000 K, 3.8] + $\left[T_{\text {eff }}(\mathrm{B}), \log g_{*}(\mathrm{~B})\right]$ for which the observed and synthetic colours are in agreement, within the uncertainties.

In order to break the degeneracies and further constrain the parameters for star B, the observed SED, which includes the available photometry and the IUE spectrum, is compared with composite Kurucz low-resolution models, built as the sum of the model for star A, [9000 K, 3.8], and those for [ $\left.T_{\text {eff }}(\mathrm{B}), \log g_{*}(\mathrm{~B})\right]$ obtained in the previous step. The models for stars $A$ and $B$, reddened with the corresponding values of $E(B-V)_{\mathrm{A}}$ and $E(B-V)_{\mathrm{B}}$

3 http://astro.wsu.edu/models/ 
Table 7. HR 10: stellar parameters and colours.

\begin{tabular}{lcc}
\hline \hline \multicolumn{3}{l}{ Parameters of the individual components } \\
\hline Parameter & Star A & Star B \\
\hline$T_{\text {eff }}(\mathrm{K})$ & $9000 \pm 100$ & $8250 \pm 100$ \\
$\log g_{*}(\mathrm{cgs})$ & $3.8 \pm 0.1$ & $4.2 \pm 0.1$ \\
$L / L_{\odot}$ (from tracks) & $64.9 \pm 10.0$ & $12.6 \pm 4.0$ \\
$L / L_{\odot}$ (from SED fit) & $57.3 \pm 2.0$ & $13.7 \pm 0.5$ \\
$M / M_{\odot}$ (from tracks) & $2.5 \pm 0.1$ & $1.8 \pm 0.1$ \\
$M / M_{\odot}($ from Eq. $(2))$ & $1.94 \pm 0.15$ & $1.62 \pm 0.13$ \\
$\left.v \sin i(\mathrm{~km} \mathrm{~s})^{-1}\right)$ & $294 \pm 9$ & $200 \pm 20$ \\
Age $(\mathrm{Myr})$ & \multicolumn{2}{c}{$530 \pm 50$} \\
$E(B-V)$ & 0.10 & 0.05 \\
\hline Observed and synthetic colours & \\
\hline Colour & Observed & Synthetic $(\mathrm{A}+\mathrm{B})$ \\
\hline$B-V$ & $+0.10 \pm 0.04$ & $+0.13 \pm 0.04$ \\
$V-H$ & $+0.40 \pm 0.04$ & $+0.40 \pm 0.04$ \\
$J-H$ & $+0.03 \pm 0.04$ & $+0.06 \pm 0.04$ \\
$J-K_{\mathrm{s}}$ & $+0.11 \pm 0.04$ & $+0.08 \pm 0.04$ \\
\hline
\end{tabular}

are scaled so that $F_{H}(\mathrm{~B}) / F_{H}(\mathrm{~A})=0.32$ at the $H$-band, and the sum is normalized to the observed SED.

A further constraint can be obtained from the HR diagram, by imposing that the point $\left[T_{\text {eff }}(\mathrm{B}), \log g_{*}(\mathrm{~B})\right]$ falls on the same isochrone as the point $[9000 \mathrm{~K}, 3.8]$. PARSEC V2.1s evolutionary tracks and isochrones ${ }^{4}$ (Bressan et al. 2012) with solar metallicity, $Z=0.017$, were used.

Once all these requirements are taken into account and fulfilled, a solution for each star is found. Table 7 shows the parameters of the individual stars, and the observed colours compared with the synthetic ones. The synthetic magnitudes of the combined pair $\mathrm{A}+\mathrm{B}$, computed by imposing $H=5.83 \pm 0.03$, are $B=6.35 \pm 0.06, V=6.22 \pm 0.05, J=5.89 \pm 0.05$, and $K_{\mathrm{s}}=5.81 \pm$ 0.06 ; both these magnitudes and the synthetic colours are in excellent agreement with the observed photometry (see Table 6).

Two sets of values for the masses and luminosities of the stars are given in Table 7. Regarding the masses, those labelled "from tracks" were derived by locating the points $\left[T_{\text {eff }}, \log g_{*}\right]$, [9000 K, 3.8] (A), and [8750, 4.2] (B), which are direct results of the above analysis, on the HR diagram $\log g_{*}-T_{\text {eff }}$, whereas those labelled "from Eq. (2)" are computed using that equation, which contains parameters obtained from the binary solution. The corresponding mass ratios are $0.72 \pm 0.05$ and $0.84 \pm 0.09$, which agree with each other within the uncertainties; there is however a discrepancy between the two values of the mass for star A, the reason could be the strong dependence of the stellar mass on $\sin ^{3} i$ (see Eq. (2)).

Regarding the luminosities, the first set of values is found by "translating" the points [9000 K, 3.8] (A) and [8750 K, 4.2] (B) into the tracks in the $L_{*} / L_{\odot}-T_{\text {eff }}$ HR diagram. Figure 12 shows the HR diagrams $\log g_{*}$ (top) and $L_{*} / L_{\odot}$ (bottom) $-T_{\text {eff }}$ with the positions of the individual components of HR 10, plotted as black dots with the corresponding error bars. The PARSEC V2.1s evolutionary tracks and isochrones are plotted in red and green, respectively. The stretch of the tracks plotted in a more intense red tone correspond to the time span where hydrogen is actively burning in the core.

The estimate of the age of the system is derived from the position of the stars in the HR diagram. Below, we give details

4 https://people.sissa.it/ sbressan/parsec.html
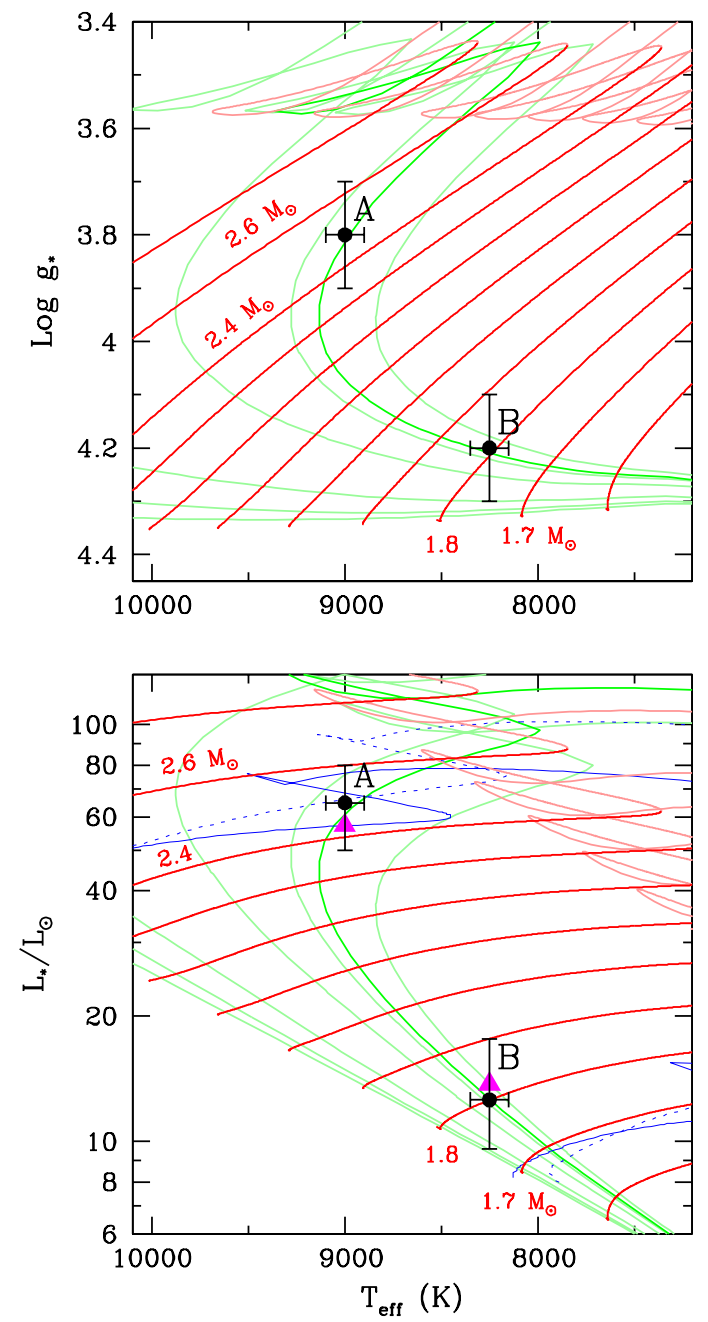

Fig. 12. HR diagrams $\log g_{*}-T_{\text {eff }}$ (top) and $L_{*} / L_{\odot}$ (bottom) and the positions of the individual components of HR 10. PARSEC V2.1s evolutionary tracks (red) and isochrones (green) are used. Isochrones correspond to 50, 100, 200, 400, 500, 530 (highlighted) and $600 \mathrm{Myr}$. Solid (dotted) blue lines in the lower panel are the evolutionary tracks with no rotation (rotation) for stars with 1.7 and $2.5 M_{\odot}$ from Ekström et al. (2012) (see Sect. 7.5). The two magenta triangles in the lower HR diagram show luminosities, described below in the main text.

about the second set of values for the luminosities in Table 7, plotted as magenta triangles in the bottom panel of Fig. 12.

Figure 13 shows the SED of HR 10 composed of the ultraviolet IUE spectrum and the photometry converted into fluxes together with the composite model solution $\mathrm{A}+\mathrm{B}$ (green) and the individual Kurucz models for stars A (red) and B (blue) reddened with values of $E(B-V) 0.10$ and 0.05 , and scaled so that $F_{H}(\mathrm{~B}) / F_{H}(\mathrm{~A})=0.32$. The plot shows the contribution of each star to the total SED very clearly. Individual luminosities for each component have been computed by dereddening each model, integrating - which provides values of $F_{\text {obs }}\left(\mathrm{erg} \mathrm{cm}^{-2} \mathrm{~s}^{-1}\right)$ at Earth - and converting the results into luminosities using the expression $L_{*}=4 \pi F_{\text {obs }} d^{2}$ where $d=145.18_{-2.45}^{+2.54}$ pc (Gaia DR2). These luminosities are shown as two magenta triangles at the bottom HR diagram of Fig. 12. The agreement between the values of the two sets of luminosities is excellent.

Figure 14 shows the comparison of the observed highresolution spectrum of HR 10 with the composite synthetic model. The spectrum is the median of 14 UVES/VLT spectra obtained on 2007-06-28, and three HARPS-N spectra obtained 


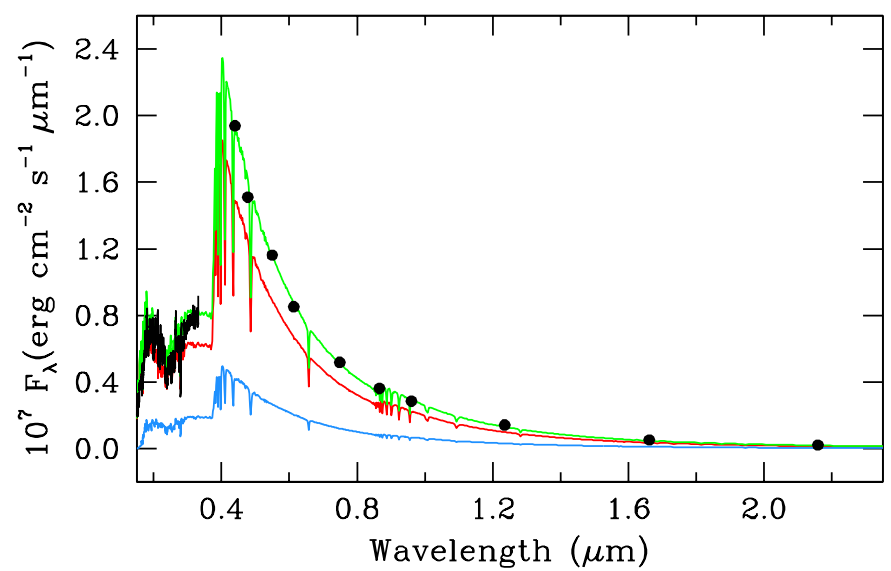

Fig. 13. Spectral energy distribution (SED) of HR 10 and the adopted solution. The observed optical and NIR photometry and the ultraviolet IUE spectrum SWP38943 + LWP18015 are plotted in black. The models for stars A and B and the combined solution are plotted in red, blue, and green, respectively.

on 2017-10-06 and 2017-10-23/24. These spectra were selected because the radial velocities of the stars are small (see Table 3), and therefore we can consider that the stars are almost in conjunction, and no substantial broadenings of the lines due to large differences between the RVs of the stars are present. Model for star A [9000 K, 3.8] was rotationally broadened with $v \sin i=$ $294 \mathrm{~km} \mathrm{~s}^{-1}$ (Mora et al. 2001). The projected rotational velocity of $200 \mathrm{~km} \mathrm{~s}^{-1}$ used for star B has been assigned after some iterations and comparison with some observed photospheric features, although this value has to be taken with caution because there is no formal way to obtain a more robust result. The depth and full width at half depth of the photospheric $\mathrm{Ca}$ II $\mathrm{K}$ profile, measured on a spline traced on the observed line are $0.40 \pm 0.02$ and $484 \mathrm{~km} \mathrm{~s}^{-1}$, respectively, whereas the values in the composite $\mathrm{A}+\mathrm{B}$ model are $0.39 \pm 0.02$ and $465 \mathrm{~km} \mathrm{~s}^{-1}$. It is interesting to point out that the bottom of the Balmer lines shows a profile that cannot be reproduced by the synthetic spectrum, that yields a slightly more rounded shape as a consequence of the high values of the projected rotational velocities of the stars.

Given the excellent agreement between the observed quantities and those extracted during the above thorough analysis, we can consider that the initial hypothesis made about the parameters $T_{\text {eff }}$ and $\log g_{*}$ for star A being close to the first estimate is consistent.

\section{Discussion}

\subsection{Interpretation of the $D, S, d$, and $s$ profiles}

We have shown that the RVs of the D and $\mathrm{S}$ narrow absorption features trace the movements of the primary and secondary components, HR 10-A and -B. The important result that immediately comes out of this is that both stars hold individual CS envelopes. Should the star have a unique, circumbinary shell, we would only observe a single narrow absorption.

In Fig. 10, already described in Sect. 6.2, we have included the RVs of components $\mathrm{d}$ and $\mathrm{s}$, plotted as pale red and blue squares. As mentioned above, these components appear redshifted with respect to D and S, and do not seem to be transient phenomena, since they are clearly apparent at any time when the difference in RVs between D and S is large enough. In addition, we show (see Fig. 5) that these weak components are also present in the CS narrow absorptions of species other than Ca II.
In what follows we carry out a quantitative analysis of components $\mathrm{d}$ and $\mathrm{s}$ for the narrow CS absorptions of the Ca II K line. Figure 15 shows the behaviour of the RVs of D, S, d, and $\mathrm{s}$ for all the observations where a four-Gaussian decomposition was feasible ${ }^{5}$. The issue is to ascertain whether or not there is a physical link between the main components $\mathrm{D}$ and $\mathrm{S}$, and the redshifted components $\mathrm{d}$ and $\mathrm{s}$.

The upper panel of Fig. 15 shows s-d plotted against S-D, that is, the difference between the radial velocities of the weak components, compared with the same quantity for the main ones. The diagonal of the diagram is plotted as a dashed green line whereas a linear fit, plotted in purple, yields a slope $0.91 \pm 0.02$ (an orthogonal fit, giving both variables a symmetric treatment, was chosen, Isobe et al. 1990). This shows that, on average, the differences in RVs between the weak components $s-d$ and the main components $\mathrm{S}-\mathrm{D}$ behave quantitatively in the same way.

Some differences are apparent when individually analysing the behaviour of components $d$ and $s$ with respect to $D$ and $\mathrm{S}$. In the middle panel of Fig. 15 the RVs of $\mathrm{d}$ (s) are plotted against the RVs of D (S) in red (blue), the slopes of the linear fits being $1.04 \pm 0.02$ and $0.81 \pm 0.02$, respectively. As a general feature, components $\mathrm{d}$ and $\mathrm{s}$ are always redshifed with respect to the main components $\mathrm{D}$ and $\mathrm{S}$; however it is interesting to note that whereas for the whole range of RVs of component $\mathrm{D}$, the distance $\mathrm{d}-\mathrm{D}$ is always within a narrow range of $10.72 \pm 1.72 \mathrm{~km} \mathrm{~s}^{-1}$ (see lower panel of Fig. 15), in the case of component $\mathrm{S}$, the mean distance $\mathrm{s}-\mathrm{S}$ is $8.29 \pm 1.79$ for positive values of $S$ and $14.18 \pm 2.47$ for negative values; the overall trend seems to be a decrease of $\mathrm{s}-\mathrm{S}$ as the RV of component $\mathrm{S}$ increases.

After this quantitative analysis, the physical link between $\mathrm{D}, \mathrm{S}, \mathrm{d}$, and s seems fairly clear, but once the transient nature of the phenomenon is discarded, the real origin of the weak components is uncertain.

\subsection{Evolutionary status of $H R 10$}

In Fig. 12 it can be seen that the components of HR 10 lie on the stretches of the tracks starting at the beginning of the MS and ending at the first turning point, where almost all hydrogen in the core is totally exhausted. During that time span, the stars are actively burning hydrogen. According to the individual PARSEC V2.1s track models, the ratio $\mathrm{H} / \mathrm{He}$ (by mass) is $\sim 0.34$ for HR 10-A and $\sim 1.36$ for HR 10-B; the initial ratio being $\sim 2.51$. The stars lie on the $\sim 530$-Myr isochrone, whereas the turning points occur at $\sim 630 \mathrm{Myr}$ and $\sim 1.6 \mathrm{Gyr}$ for A and B, respectively, away in both cases of the red giant branch phase, especially for star B. Taking all this into account, HR 10-A would be in an evolutionary stage close to subgiant, where HR 10-B is still very close to the MS. Since a star remains on the main sequence as long as there is hydrogen in the core that it can fuse into helium, we can consider that HR 10 is still in the MS, although speaking more precisely, it would be a very early post-MS binary.

\subsection{Binarity and shell/discs}

The value of $a_{\text {tot }}(10.58$ mas, Table 5$)$ at the distance to HR 10 ( $145.18 \mathrm{pc}$ ) is equivalent to $\sim 3.08 \mathrm{au}$, which implies, according to the value of the masses and eccentricities, a minimum separation between the stars - when both components are at the corresponding pericentres - of $\sim 2.39$ au. The simple sketch of Fig. 16

5 To avoid a tedious notation, we use the nomenclature $\mathrm{S}-\mathrm{D}, \mathrm{s}-\mathrm{d}$, $\mathrm{d}-\mathrm{D}$, and $\mathrm{s}-\mathrm{S}$ to express the differences in radial velocities $\mathrm{RV}(\mathrm{S})-$ $\mathrm{RV}(\mathrm{D}), \mathrm{RV}(\mathrm{s})-\mathrm{RV}(\mathrm{d}), \mathrm{RV}(\mathrm{d})-\mathrm{RV}(\mathrm{D})$ and $\mathrm{RV}(\mathrm{s})-\mathrm{RV}(\mathrm{S})$, respectively. 


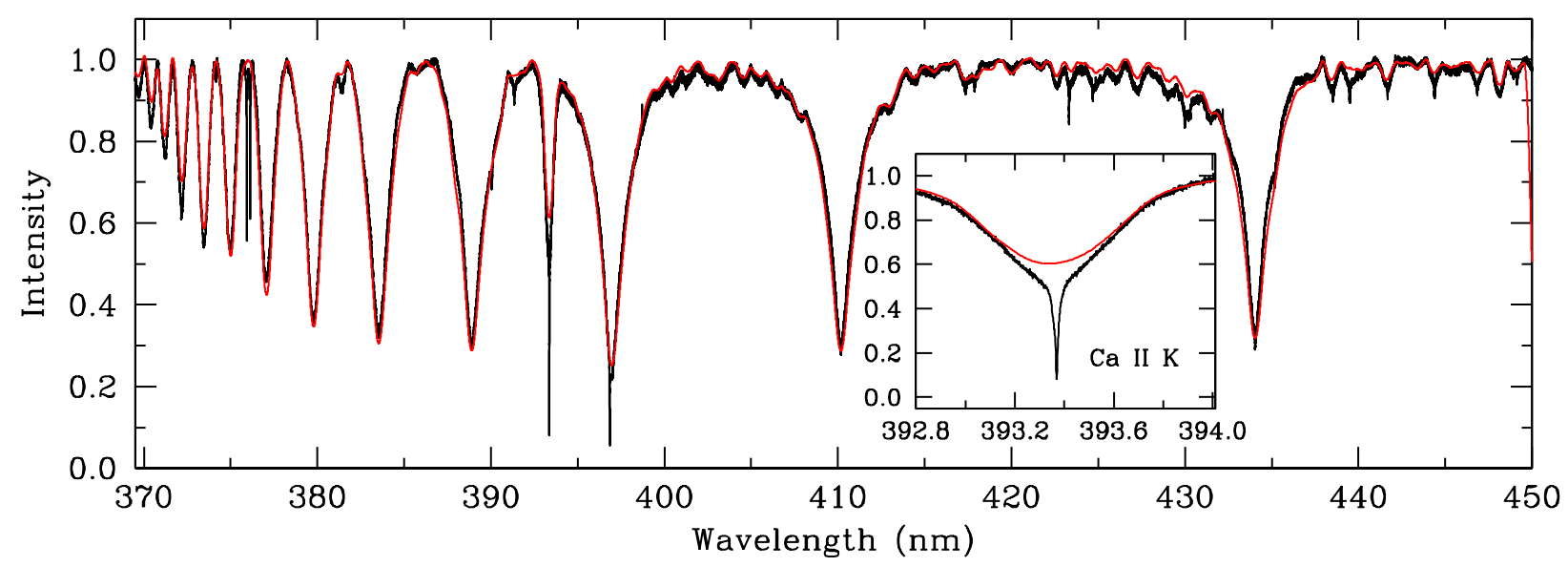

Fig. 14. Spectrum of HR 10 (black) and the spectral fit (red) computed for the composite model for the stellar parameters given in Table 7. The inset shows a blow-up of the Ca II K profile and the fit. The main narrow features that are not reproduced by the synthetic model - which only accounts for the photospheric spectrum - are the Ti II lines at 375.93 and $376.13 \mathrm{~nm}$, and the Ca II HK lines at 393.37 and $396.85 \mathrm{~nm}$ (see Sect. 5).

illustrates that particular configuration, showing the orbits of HR 10-A and -B around the centre of mass as they are observed and seen pole-on, deprojecting the orbits using the inclination, $i\left(93.34^{\circ}\right.$, Table 5). That minimum separation is a small value, and leaving aside a dynamical and evolutionary study of the binary, which is out of the scope of this paper, it is remarkable to point out that (i) both stars maintain their individual envelopes or shells, and (ii) the absence of a stable narrow absorption component at the systemic velocity of the system implies that no circumbinary envelope is present. Some studies on binarity and discs have been carried out in other contexts; for example, Herbig AeBe stars (see e.g. Duchêne 2015), T Tauri stars (Harris et al. 2012), and debris discs around solar-type and intermediatemass stars (Rodriguez \& Zuckerman 2012). However, to the best of our knowledge, there have been no studies devoted to the MS or early post-MS binary systems with shells. Therefore, this system is the first of its class to be discovered and studied.

\subsection{TESS photometry: rotation and pulsations}

For the sake of completeness, we would like to mention the observations of HR 10 obtained by the Transit Exoplanet Survey Satellite mission (TESS) during Sector 2 in Camera 1 and CCD 3 (TIC 289592423). The photometric observations span a total of 27.4 days with a short cadence of $2.15 \mathrm{~min}$. The data were downloaded from the Mikulski Archive for Space Telescopes 6 (MAST), including the extracted light curve by the Science Processing Operations Center (SPOC) pipeline. The median photometric uncertainty per datapoint for the pre-search data conditioning simple aperture photometry (known as PDCSAP) is 140 parts per million. Given the large pixels and apertures used to extract the light curve, the binary system is not resolved by TESS. In Fig. 17 the light curve shows clear periodic photometric variations with $\sim 5$ mmag semi-amplitude. As an example of how the variability pattern repeats itself, the observations corresponding to $\sim 6$ days at the end of the observing window highlighted in red - have been shifted in time by approximately -19.630 days and superimposed on the original data at the beginning of the observing run, fine-tuning the shift and causing the maxima and minima to coincide. The residuals between the shifted and the original data are less than $5.0 \times 10^{-3}$, the average of the absolute values being $1.0 \times 10^{-3}$. The analysis

6 http://archive.stsci.edu of these data, which include effects of rotation, pulsation, and gravitational darkening from the two components of HR 10, will be presented in another paper (Barceló-Forteza et al., in prep.).

\subsection{Caveats}

Finally, we would like to point out explicitly some caveats that must be taken into account when using and interpreting the results presented here.

The projected rotational velocities of the stars are large, and therefore they must produce geometrical distortions which imply an oblateness of the objects, which in turn results in a larger (smaller) gravity and temperature at the poles (equator), the so-called gravitational darkening. Further phenomena, such as differential rotation, prevent us from assigning a single temperature, gravity, or rotation velocity to the stars (see e.g. Zorec et al. 2017, and references therein).

Even if each component can be characterized by a single magnitude and colour, its position in colour-magnitude diagrams, and hence in the HR diagram, when translating magnitudes and colours into luminosities and temperatures, can be altered by rotation (see e.g. Bastian \& de Mink 2009).

The PARSEC V2.1s tracks used during the computation of the stellar parameters do not include rotation. For comparison purposes we plotted the evolutionary tracks with no rotation (rotation) for stars with 1.7 and $2.5 M_{\odot}$ and $Z=0.014$ from Ekström et al. (2012) as solid (dotted) blue lines in the lower HR diagram of Fig. 12. This grid only includes models for stars with $1.5,1.7,2.0$, and $2.5 M_{\odot}$, in the range of interest for this work, an overly scarce sampling for our purposes, and are computed for rotation velocities that do not correspond to those of HR 10 . Therefore, we were not able to use them for the analysis. In any case the deviation with respect to the PARSEC V2.1s tracks does not seem to be dramatic.

\section{Conclusions}

Here we present a complete analysis of the star HR 10 in the context of a large programme aimed at detecting and monitoring variable metallic features superimposed on the photospheric lines that could be attributed to exocometary events (Rebollido et al. 2019). HR 10 was singled out because of its peculiar variability, the availability of a large amount of high-resolution spectra in archives and publications, and the hints of a first 

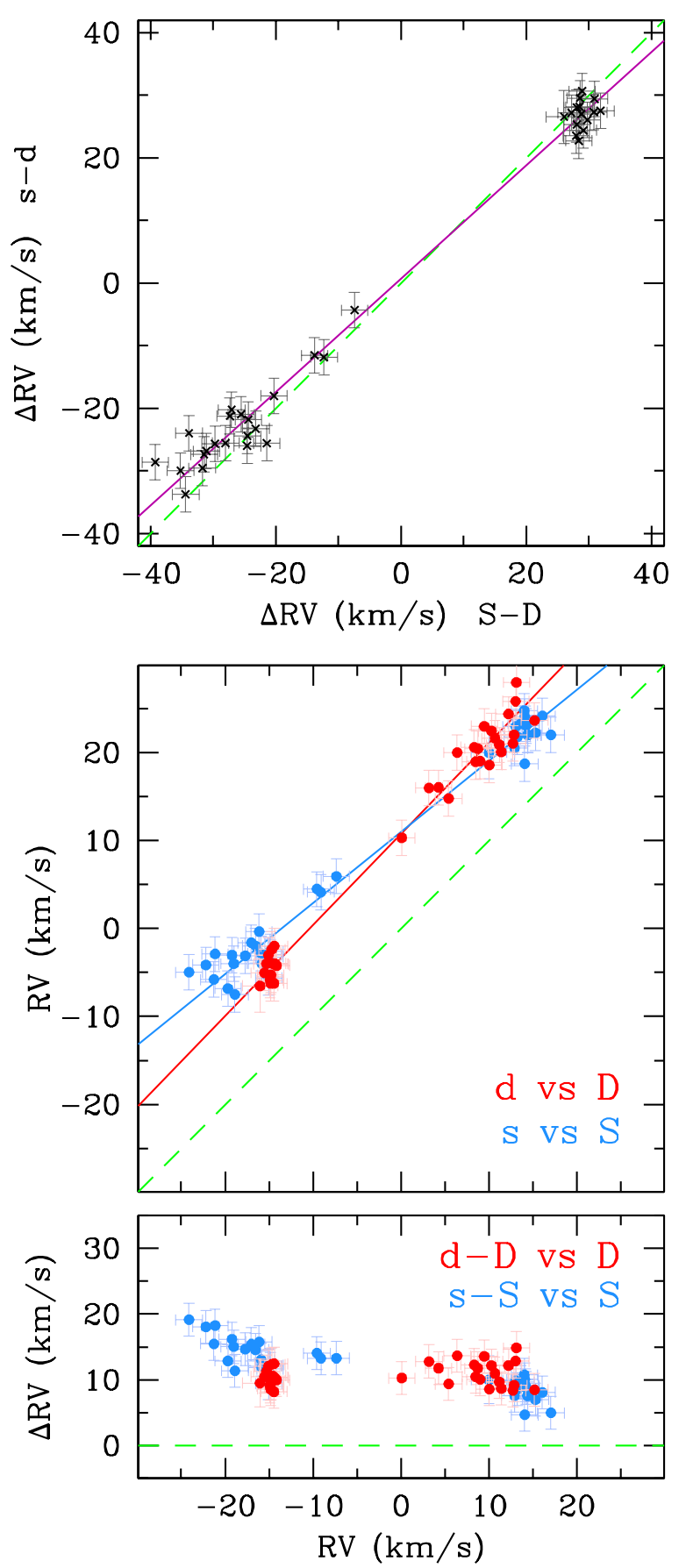

Fig. 15. Top: difference between the RVs of components $s$ and $\mathrm{d}$ for each observation, $s-d$, plotted against the difference between the corresponding RV of components S and D, S-D. Middle: RV of components $\mathrm{d}$ and s, plotted against the corresponding RV of components D and S; the dashed green line is the 1:1 relationship. Bottom: differences in RVs $\mathrm{d}-\mathrm{D}$ and $\mathrm{s}-\mathrm{S}$ plotted against $\mathrm{D}$ and $\mathrm{S}$, respectively.

interferometric PIONIER/VLTI observation obtained in 2014 that suggested that HR 10 could be a binary. Dedicated campaigns, both with that instrument and with spectrographs in several telescopes, together with all the archival material have allowed us to carry out a thorough study whose main results can be summarised as follows.

1. Four interferometric PIONIER/VLT observations have provided solid evidence that HR 10 is a binary, the contrast between the components being $\sim 32 \%$ in $H$-band.

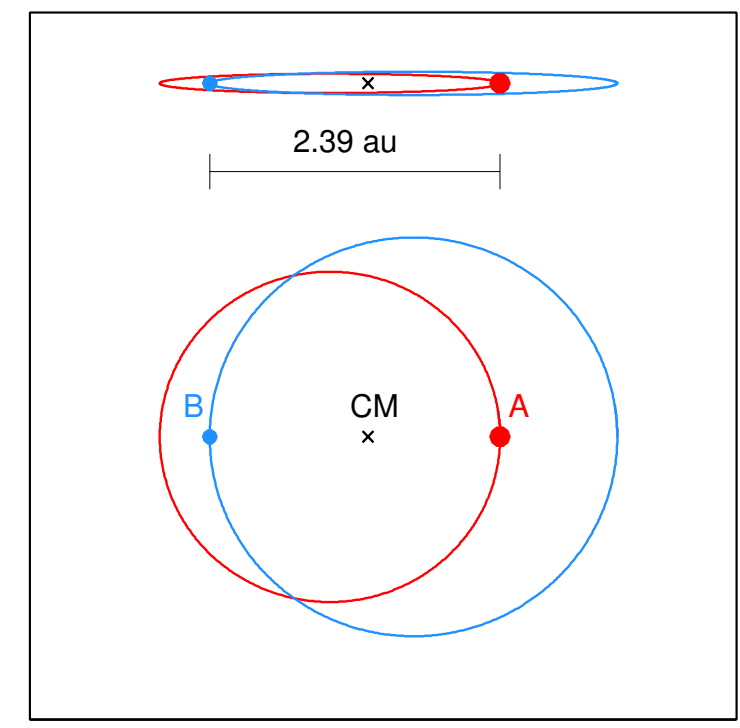

Fig. 16. Simple sketch of the orbits of HR 10-A and -B around the centre of mass of the system plotted at scale as they are observed (top) and deprojected onto a plane using the value of the inclination, $i$, from Table 5. The stars have been located at the corresponding pericentres, i.e. the points where the distance between components $\mathrm{A}$ and $\mathrm{B}$ is a minimum $(\sim 2.39 \mathrm{au})$.

2. The analysis of more that $32 \mathrm{yr}$ of high-resolution spectroscopic observations from archives, publications, and since 2015, from our dedicated campaigns, shows that narrow CS absorption features are present in at least 24 metallic lines of Ca II, Ti II, and Fe II.

3. Particular attention has been paid in this work to the strong Ca II K CS narrow absorption features, which can be decomposed into two main components, labelled "D" and "S" - for "deep" and "shallow". These components show a periodic behaviour, with component D moving to the blue (red) as component $\mathrm{S}$ moves to the red (blue), crossing each other at certain times; this pattern being kept over decades.

4. In those situations where the separation in RVs of components $\mathrm{D}$ and $\mathrm{S}$ is large, two additional weaker components, labelled " $d$ " and "s" are very apparent, each one to the red side of components D and S, respectively. These weaker d and $s$ absorption features seem to accompany the main $\mathrm{D}$ and $\mathrm{S}$ in their periodic movements and not only appear in the Ca II K narrow absorptions, but also in lines of other species, like Ti II and Fe II.

5. Each star holds its individual shell or envelope. The detailed analysis of the time evolution of components D and S shows that they trace the orbit of each individual star in the binary. This is strongly confirmed by the spectrometric binary solution. The orbital period is $P_{\text {orb }}=747.6$ days, the mass ratio $q=M_{\mathrm{B}} / M_{\mathrm{A}} \simeq 0.72-0.84$, and the eccentricity of the orbits is $e \simeq 0.23$.

6. The complete orbital solution was obtained using the results of the RV analysis and the four PIONIER/VLTI astrometric points. This allowed us to compute the inclination of the system, $i=93.34^{\circ}$ and the total semimajor axis, $a_{\mathrm{tot}}=a_{\mathrm{A}}+$ $a_{\mathrm{B}}=10.58$ mas, which at the distance to HR $10(145.18 \mathrm{pc})$ is equivalent to $\sim 3.08 \mathrm{au}$; this implies a minimum distance between the stars - when both are located at their pericentre - of $\sim 2.39 \mathrm{au}$, a remarkably small value.

7. Stellar parameters for HR 10-A and -B were estimated making use of observable quantities - photometry, optical 


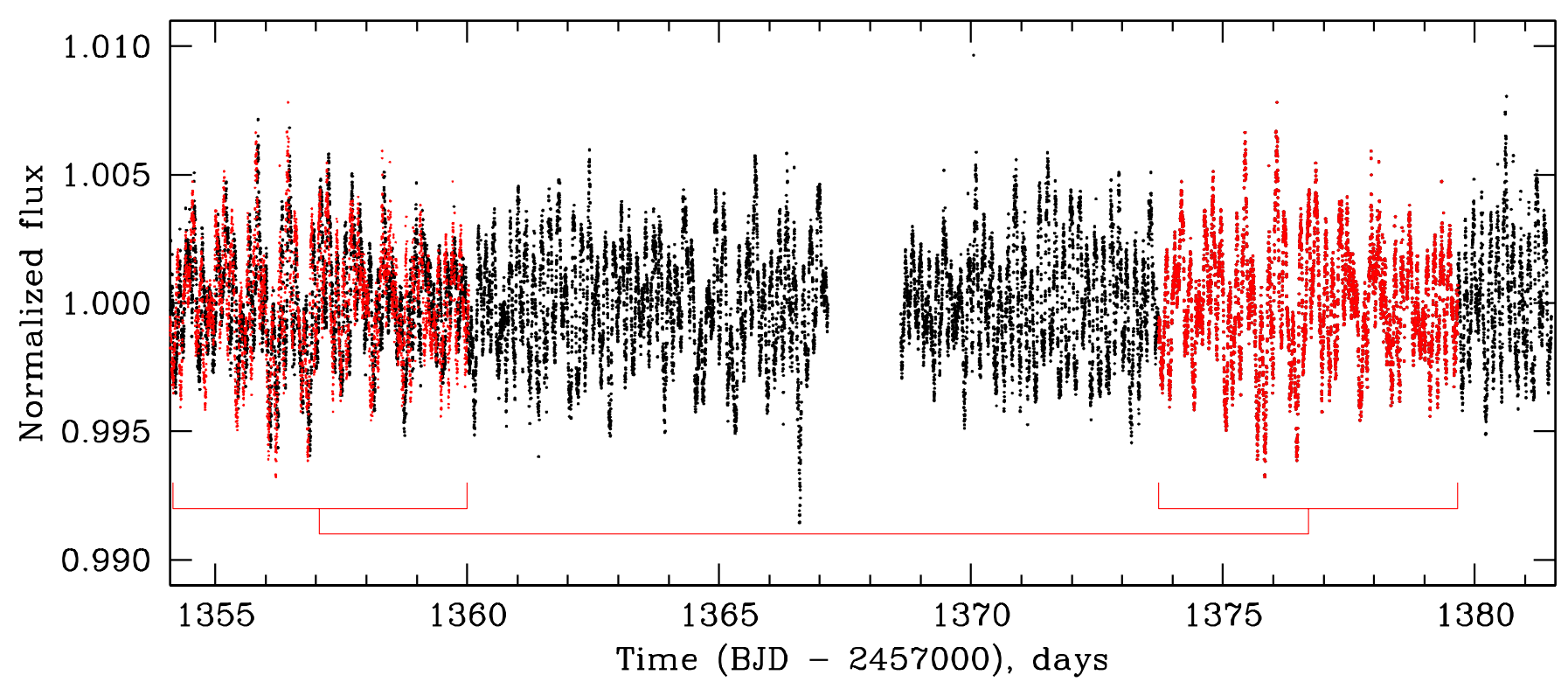

Fig. 17. TESS photometric time series, showing the very short-time variability of the HR 10 system. As an example of the repeatibility of the pattern, the observations between days 1373.72 and 1379.66 (in BJD-2 457 000) highlighted in red have been shifted to the interval 1354.11-1360.05, where they are overplotted in red on the original set of data. See text for details.

and ultraviolet spectra, and the constraint imposed by the PIONIER observations - and synthetic low- and highresolution spectra and evolutionary tracks.

8. To our knowledge, this is the first case studied of a (slightly off)-MS binary where both components have individual envelopes or shells. The current analysis does not show any variability in the CS components that could be attributed to exocometary events. The absence of a narrow absorption component at the systemic velocity rules out the presence of a circumbinary envelope.

As a final remark, we would like to explicitly mention the fact that there is a chance that other stars that exhibit variability attributed to FEB phenomena might actually be binaries and behave in a way qualitatively similar to HR 10. Marion et al. (2014) estimated that when using interferometry and singleaperture imaging, about half the population of nearby A-type stars could be resolved as binaries, and suggested that a number of them remain undetected. In the case of HR 10, the key point that drove the whole analysis presented in this paper was the first PIONIER observation obtained in 2014 that showed strong evidence that the star was a binary.

In a generic case, should the interferometric observations not be feasible, spectroscopy alone would be able to unveil the binarity, provided that observations obtained during a well-sampled and long time interval can be collected; the binarity would show up in the structure of the photospheric lines provided the projected rotation velocities of the stars were low enough, and/or in the periodic shifts of the narrow absorption component (components), if it (they) were present and found to show a similar shape during timescales long enough to rule out the FEB scenario. Otherwise, if the orbital period of the binary is of the order of months or years, the analysis of the variability of the narrow absorptions during shorter intervals (days or weeks) can be misinterpreted as originating from an exocometary event.

Acknowledgements. We are grateful to Prof. Barry Welsh, who refereed this paper, for his report and comments. The authors also acknowledge Prof. Lynne Hillenbrand for her valuable suggestions, scientific discussion, and for coordinating the reduction of the HIRES archival spectra, and Prof. René Oudmaijer, Dr Antonio Garufi, and Dr Sebastià Barceló for their comments; we also thank Dr Valentin Christiaens for obtaining the 2014 PIONIER observations, Daniela Paz
Iglesias for covering the FEROS 2015 campaign, and Dr Trifon Trifonov for his advice during the reduction of some spectroscopic data. This work is partially based on observations made with the Nordic Optical Telescope (NOT), operated by the Nordic Optical Telescope Scientific Association (NOTSA), the Italian Telescopio Nazionale Galileo (TNG), operated by the Fundación Galileo Galilei of the Istituto Nazionale di Astrofisica (INAF), and the Mercator Telescope, operated by the Flemmish Community; all three located at the Spanish Observatorio del Roque de los Muchachos of the Instituto de Astrofísica de Canarias on the island of La Palma; also on observations obtained with the MPIA-ESO/2.2$\mathrm{m}$ telescope operated at La Silla Observatory by the Max-Planck-Institut für Astronomie, and observations collected at the European Southern Observatory with the Very Large Telescope Interferometer (VLTI) operated at Cerro Paranal, under ESO programmes 093.C-0712(B), 099.C-2015(A) and 0101.C0182(B). This paper also includes data taken with the 2.7-m Harlan J. Smith Telescope at McDonald Observatory of The University of Texas at Austin; we thank David Doss at McDonald Observatory for his valuable assistance in obtaining the high resolution spectra. Data acquired at the Anglo-Australian Telescope (AAT) have been also used; we acknowledge the traditional owners of the land on which the AAT stands, the Gamilaraay people, and pay our respects to elders past and present; we thank Stuart Ryder for his assistance at the AAT. Data from the ESO Science Archive Facility, programmes 079.C-0789 and 094.C-0946, have been used. This research has made use of the Keck Observatory Archive (KOA), which is operated by the W.M. Keck Observatory and the NASA Exoplanet Science Institute (NExScI), under contract with the National Aeronautics and Space Administration; data from programmes U101Hr (PI: G. Marcy) and C328Hr (PI: J. Johnson) were used. C.E., G.M., B.M., I.R., and E.V. are supported by Spanish grant AYA 2014-55840-P; H.C. acknowledges funding from the ESA Research Fellowship Programme; J.J.E.K. acknowledges support from the Academy of Finland grant 295114, and thanks the Universidad Autónoma de Madrid staff for their hospitality; the research of I.M. is funded by a Talento Fellowship (2016-T1/TIC-1890, Government of Comunidad Autónoma de Madrid, Spain). This work has made use of data from the European Space Agency (ESA) missions International Ultraviolet Explorer (IUE) (archive http://sdc.cab.inta-csic.es/ines/), and Gaia (https://www.cosmos.esa.int/gaia), processed by the Gaia Data Processing and Analysis Consortium (DPAC, https://www.cosmos.esa.int/ web/gaia/dpac/consortium). This research has made use of the SIMBAD database, operated at CDS, Strasbourg, France.

\section{References}

Absil, O., di Folco, E., Mérand, A., et al. 2006, in Visions for Infrared Astronomy, Instrumentation, Mesure, Metrologie, eds. V. Coudé du Foresto, D. Rouan, \& G. Rousset, 251

Absil, O., Le Bouquin, J. B., Berger, J. P., et al. 2011, A\&A, 535, A68 Absil, O., Defrère, D., Coudé du Foresto, V., et al. 2013, A\&A, 555, A104 Abt, H. A. 2008, ApJS, 174, 499 
Aller, L. H., Appenzeller, I., Baschek, B., et al., eds. 1982, Landolt-Börnstein: Numerical Data and Functional Relationships in Science and Technology New Series, Group 6 Astronomy and Astrophysics, Stars and Star Clusters (Berlin: Springer), 2, 54

Armitage, P. J. 2015, ArXiv e-prints [arXiv:1509.06382]

Backman, D. E., \& Paresce, F. 1993, in Protostars and Planets III, eds. E. H. Levy \& J. I. Lunine (Tucson, AZ: University of Arizona Press), 1253

Bastian, N., \& de Mink S. E. 2009, MNRAS, 398, L11

Bessell, M. S. 1979, PASP, 91, 589

Beust, H., Lagrange-Henri, A. M., Madjar, A. V., \& Ferlet, R. 1990, A\&A, 236, 202

Beust, H., Vidal-Madjar, A., \& Ferlet, R. 1991, A\&A, 247, 505

Beuther, H., Klessen, R. S., Dullemond, C. P., \& Henning, T. 2014, Protostars and Planets VI (Tucson, AZ: University of Arizona Press)

Bressan, A., Marigo, P., Girardi, L., et al. 2012, MNRAS, 427, 127

Castelli, F., \& Kurucz, R. L. 2003, Modelling of Stellar Atmospheres, eds. N. Piskunov, W. W. Weiss, \& D. F. Gray, IAU Symp., 210, A20

Chambers, K. C., Magnier, E. A., Metcalfe, N., et al. 2016, ArXiv e-prints [arXiv: 1612.05560$]$

Cheng, K.-P., Grady, C. A., \& Bruhweiler, F. C. 1991, ApJ, 366, L87

Cohen, M., Wheaton, W. A., \& Megeath, S. T. 2003, AJ, 126, 1090

Cutri, R. M., Skrutskie, M. F., van Dyk, S., et al. 2003, VizieR Online Data Catalog: II/246

Duchêne, G. 2015, Ap\&SS, 355, 291

Eiroa, C., Rebollido, I., Montesinos, B., et al. 2016, A\&A, 594, L1

Ekström, S., Georgy, C., Eggenberger, P., et al. 2012, A\&A, 537, A146

Ertel, S., Absil, O., Defrère, D., et al. 2014, A\&A, 570, A128

Ertel, S., Defrère, D., Absil, O., et al. 2016, A\&A, 595, A44

Ertel, S., Defrère, D., Hinz, P., et al. 2018, AJ, 155, 194

Faramaz, V., Ertel, S., Booth, M., Cuadra, J., \& Simmonds, C. 2017, MNRAS, 465,2352

Ferlet, R., Hobbs, L. M., \& Madjar, A. V. 1987, A\&A, 185, 267

Foreman-Mackey, D., Hogg, D. W., Lang, D., \& Goodman, J. 2013, PASP, 125,306

Gaia Collaboration 2018, VizieR Online Data Catalog: I/345

Grady, C. A., Brown, A., Welsh, B., et al. 2018, AJ, 155, 242

Harris, R. J., Andrews, S. M., Wilner, D. J., \& Kraus, A. L. 2012, ApJ, 751, 115

Heinrichsen, I., Walker, H. J., Klaas, U., Sylvester, R. J., \& Lemke, D. 1999, MNRAS, 304, 589

Hobbs, L. M., Vidal-Madjar, A., Ferlet, R., Albert, C. E., \& Gry, C. 1985, ApJ, 293, L29
Howarth, I. D., Murray, J., Mills, D., \& Berry, D. S. 2004, Starlink User Note, 50

Isobe, T., Feigelson, E. D., Akritas, M. G., \& Babu, G. J. 1990, ApJ, 364, 104 Ivezić, Ž., Connolly, A., Vanderplas, J., \& Gray, A. 2014, Statistics, Data Mining and Machine Learning in Astronomy (Princeton: Princeton University Press)

Jaschek, C., Jaschek, M., Andrillat, Y., \& Egret, D. 1991, A\&A, 252, 229

Kausch, W., Noll, S., Smette, A., et al. 2015, A\&A, 576, A78

Kiefer, F., Lecavelier des Etangs, A., Boissier, J., et al. 2014, Nature, 514, 462

Kondo, Y., \& Wamsteker, W. 1987, Exploring the Universe with the IUE Satellite, Astrophysics and Space Science Library (Dordrecht: Springer), 129

Kurucz, R. 1993, SYNTHE Spectrum Synthesis Programs and Line Data. Kurucz CD-ROM No. 18. (Cambridge, MA: Smithsonian Astrophysical Observatory), 18

Lagrange-Henri, A. M., Beust, H., Ferlet, R., Hobbs, L. M., \& Madjar, A. V. 1990, A\&A, 227, L13

Le Bouquin, J.-B., Berger, J.-P., Lazareff, B., et al. 2011, A\&A, 535, A67

Marion, L., Absil, O., Ertel, S., et al. 2014, A\&A, 570, A127

Marino, S., Matrà, L., Stark, C., et al. 2016, MNRAS, 460, 2933

Matthews, B. C., Krivov, A. V., Wyatt, M. C., Bryden, G., \& Eiroa, C. 2014, Protostars and Planets VI (Tucson, AZ: University of Arizona Press), 521

Mede, K., \& Brandt, T. D. 2017, AJ, 153, 135

Mennesson, B., Millan-Gabet, R., Serabyn, E., et al. 2014, ApJ, 797, 119

Montesinos, B., Eiroa, C., Mora, A., \& Merín, B. 2009, A\&A, 495, 901

Moór, A., Henning, T., Juhász, A., et al. 2015, ApJ, 814, 42

Mora, A., Merín, B., Solano, E., et al. 2001, A\&A, 378, 116

Olofsson, J., Juhász, A., Henning, T., et al. 2012, A\&A, 542, A90

Rebollido, I., Eiroa, C., Montesinos, B., et al. 2018, A\&A, 614, A3

Rebollido, I., Eiroa, C., Montesinos, B., et al. 2019, A\&A, submitted

Redfield, S., Kessler-Silacci, J. E., \& Cieza, L. A. 2007, ApJ, 661, 944

Roberge, A., \& Weinberger, A. J. 2008, ApJ, 676, 509

Rodriguez, D. R., \& Zuckerman, B. 2012, ApJ, 745, 147

Smette, A., Sana, H., Noll, S., et al. 2015, A\&A, 576, A77

Wang, S., \& Chen, X. 2019, ApJ, 877, 116

Welsh, B. Y., \& Montgomery, S. L. 2015, Adv. Astron., 2015, 980323

Welsh, B. Y., Craig, N., Crawford, I. A., \& Price, R. J. 1998, A\&A, 338, 674

Worthey, G., \& Lee, H.-c. 2011, ApJS, 193, 1

Wright, C. O., Egan, M. P., Kraemer, K. E., \& Price, S. D. 2003, AJ, 125 , 359

Zechmeister, M., \& Kürster, M. 2009, A\&A, 496, 577

Zorec, J., Rieutord, M., Espinosa Lara, F., et al. 2017, A\&A, 606, A32

Zuckerman, B., \& Song, I. 2012, ApJ, 758, 77 